Supporting Information for:

\title{
Total Synthesis of Buergerinin F and Buergerinin G
}

\author{
Jeong-Seok Han and Todd L. Lowary \\ Department of Chemistry, The Ohio State University \\ Columbus, Ohio 43210 \\ lowary.2@osu.edu
}

\section{Table of Contents}

Table S1. Comparison of ${ }^{1} \mathrm{H}$ - and ${ }^{13} \mathrm{C}-\mathrm{NMR}$ data for synthetic 1 vs those reported for the material isolated from Scrophularia buergeriana.

Table S2. Comparison of ${ }^{1} \mathrm{H}$ - and ${ }^{13} \mathrm{C}-\mathrm{NMR}$ data for synthetic 2 vs those reported for the material isolated from Scrophularia buergeriana.

NMR Spectra of 1ロ16 
Table S1. Comparison of ${ }^{1} \mathrm{H}$ - and ${ }^{13} \mathrm{C}-\mathrm{NMR}$ data for synthetic $\mathbf{1}$ vs those reported for the material isolated from Scrophularia buergeriana. ${ }^{a}$

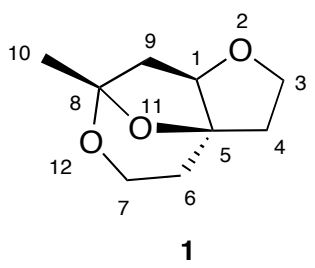

\begin{tabular}{c|c|c|c|c}
\hline Position & \multicolumn{2}{|c|}{${ }^{1} \mathbf{H}^{\mathrm{b}}$} & \multicolumn{2}{c}{${ }^{13} \mathbf{C}^{\mathrm{c}}$} \\
\hline & Synthetic & Isolated & Synthetic & Isolated \\
\hline 1 & $4.37(1 \mathrm{H}, \mathrm{dd}, 6.7,2.1 \mathrm{~Hz})$ & $4.47(1 \mathrm{H}, \mathrm{dd}, 6.7,2.0 \mathrm{~Hz})$ & 84.4 & 84.5 \\
\hline 3 & $4.10(1 \mathrm{H}, \mathrm{td}, 8.4,6.4 \mathrm{~Hz})$ & $4.10(1 \mathrm{H}, \mathrm{td}, 8.6,6.4 \mathrm{~Hz})$ & 68.8 & 68.8 \\
\hline & $3.93(1 \mathrm{H}, \mathrm{td}, 8.4,3.3 \mathrm{~Hz})$ & $3.95(1 \mathrm{H}, \mathrm{td}, 8.6,3.2 \mathrm{~Hz})$ & & \\
\hline 4 & $2.11(1 \mathrm{H}, \mathrm{ddd}, 13.2,6.4$, & $2.12(1 \mathrm{H}, \mathrm{ddd}, 13.1,6.4$, & 36.1 & 36.2 \\
& $3.4 \mathrm{~Hz})$ & $3.2 \mathrm{~Hz})$ & & \\
\hline & $1.82(1 \mathrm{H}, \mathrm{td}, 13.3,8.8 \mathrm{~Hz})$ & $1.85(1 \mathrm{H}, \mathrm{td}, 13.1,8.6 \mathrm{~Hz})$ & & \\
\hline 5 & & & 91.5 & 91.6 \\
\hline 6 & $2.24(1 \mathrm{H}, \mathrm{td}, 12.8,6.4 \mathrm{~Hz})$ & $2.25(1 \mathrm{H}, \mathrm{td}, 12.9,6.3 \mathrm{~Hz})$ & 30.6 & 30.7 \\
\hline & $1.39(1 \mathrm{H}, \mathrm{dd}, 13.0,3.8 \mathrm{~Hz})$ & $1.40(1 \mathrm{H}, \mathrm{dd}, 12.9,3.8 \mathrm{~Hz})$ & & \\
\hline 7 & $3.97(1 \mathrm{H}, \mathrm{dd}, 12.2,6.1 \mathrm{~Hz})$ & $4.00(1 \mathrm{H}, \mathrm{dd}, 12.2,6.3 \mathrm{~Hz})$ & 61.3 & 61.4 \\
\hline & $3.69(1 \mathrm{H}, \mathrm{td}, 12.2,3.9 \mathrm{~Hz})$ & $3.70(1 \mathrm{H}, \mathrm{td}, 12.2,3.8 \mathrm{~Hz})$ & & \\
\hline 8 & & & 108.5 & 108.6 \\
\hline 9 & $2.37(1 \mathrm{H}, \mathrm{dd}, 14.3,6.7 \mathrm{~Hz})$ & $2.42(1 \mathrm{H}, \mathrm{dd}, 14.4,6.7 \mathrm{~Hz})$ & 44.7 & 44.8 \\
\hline & $2.02(1 \mathrm{H}, \mathrm{dd}, 14.3,2.2 \mathrm{~Hz})$ & $2.05(1 \mathrm{H}, \mathrm{dd}, 14.4,2.0 \mathrm{~Hz})$ & & \\
\hline 10 & $1.49(3 \mathrm{H}, \mathrm{s})$ & $1.50(3 \mathrm{H}, \mathrm{s})$ & 24.2 & 24.3 \\
\hline
\end{tabular}

a. NMR spectrum recorded in $\mathrm{CHCl}_{3}$ at $400 \mathrm{MHz}\left({ }^{1} \mathrm{H}\right)$ or $100.6 \mathrm{MHz}\left({ }^{13} \mathrm{C}\right)$. ${ }^{\text {b. Chemical }}$ shift in ppm. In parentheses are the signal integration and coupling constants, in $\mathrm{Hz}$. c.Chemical shift in ppm. 
Table S2. Comparison of ${ }^{1} \mathrm{H}$ - and ${ }^{13} \mathrm{C}-\mathrm{NMR}$ data for synthetic 2 vs those reported for the material isolated from Scrophularia buergeriana. ${ }^{a}$

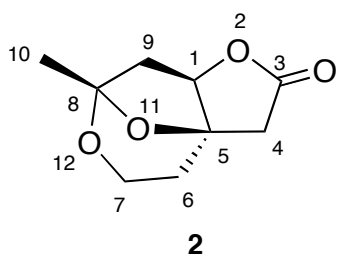

\begin{tabular}{c|c|c|c|c}
\hline Position & \multicolumn{2}{|c|}{${ }^{1} \mathbf{H}^{\mathrm{b}}$} & \multicolumn{2}{c}{${ }^{13} \mathbf{C}^{\mathrm{c}}$} \\
\hline & Synthetic & Isolated & Synthetic & Isolated \\
\hline 1 & $4.85(\mathrm{dd}, 1 \mathrm{H}, 6.8,1.8 \mathrm{~Hz})$ & $4.75(1 \mathrm{H}, \mathrm{dd}, 6.4,1.3 \mathrm{~Hz})$ & 84.2 & 84.2 \\
\hline 3 & & & 174.6 & 174.5 \\
\hline 4 & $2.75(1 \mathrm{H}, \mathrm{d}, 18.0 \mathrm{~Hz})$ & $2.75(1 \mathrm{H}, \mathrm{d}, 18.0 \mathrm{~Hz})$ & 39.3 & 39.2 \\
\hline & $2.53(1 \mathrm{H}, \mathrm{d}, 18.0 \mathrm{~Hz})$ & $2.55(1 \mathrm{H}, \mathrm{d}, 18.0 \mathrm{~Hz})$ & & \\
\hline 5 & & & 85.9 & 85.9 \\
\hline 6 & $2.27(1 \mathrm{H}, \mathrm{td}, 12.9,6.5 \mathrm{~Hz})$ & $2.25(1 \mathrm{H}, \mathrm{td}, 12.9,6.4 \mathrm{~Hz})$ & 29.8 & 29.7 \\
\hline & $1.47(1 \mathrm{H}, \mathrm{dd}, 12.9,3.7 \mathrm{~Hz})$ & $1.46(1 \mathrm{H}, \mathrm{dd}, 12.9,3.8 \mathrm{~Hz})$ & & \\
\hline 7 & $4.01(1 \mathrm{H}, \mathrm{dd}, 12.2,6.4 \mathrm{~Hz})$ & $4.00(1 \mathrm{H}, \mathrm{dd}, 12.3,6.4 \mathrm{~Hz})$ & 60.0 & 59.9 \\
\hline & $3.69,(1 \mathrm{H}, \mathrm{td}, 12.4,3.8 \mathrm{~Hz})$ & $3.70(1 \mathrm{H}, \mathrm{td}, 12.3,3.8 \mathrm{~Hz})$ & & \\
\hline 8 & & & 107.8 & 107.7 \\
\hline 9 & $2.50(\mathrm{dd}, 1 \mathrm{H}, 14.9,6.8 \mathrm{~Hz})$ & $2.49(1 \mathrm{H}, \mathrm{dd}, 14.8,6.4 \mathrm{~Hz})$ & 42.7 & 42.7 \\
\hline & $2.21(\mathrm{H}, \mathrm{dd}, 14.8,1.8 \mathrm{~Hz})$ & $2.20(1 \mathrm{H}, \mathrm{dd}, 14.8,1.3 \mathrm{~Hz})$ & & \\
\hline 10 & $1.50(3 \mathrm{H}, \mathrm{s})$ & $1.50(3 \mathrm{H}, \mathrm{s})$ & 23.9 & 23.9 \\
\hline
\end{tabular}

a. $\mathrm{NMR}$ spectrum recorded in $\mathrm{CHCl}_{3}$ at $400 \mathrm{MHz}\left({ }^{1} \mathrm{H}\right)$ or $100.6 \mathrm{MHz}\left({ }^{13} \mathrm{C}\right)$. ${ }^{\text {b. Chemical }}$ shift in ppm. In parentheses are the signal integration and coupling constants, in $\mathrm{Hz}$. c. Chemical shift in ppm. 

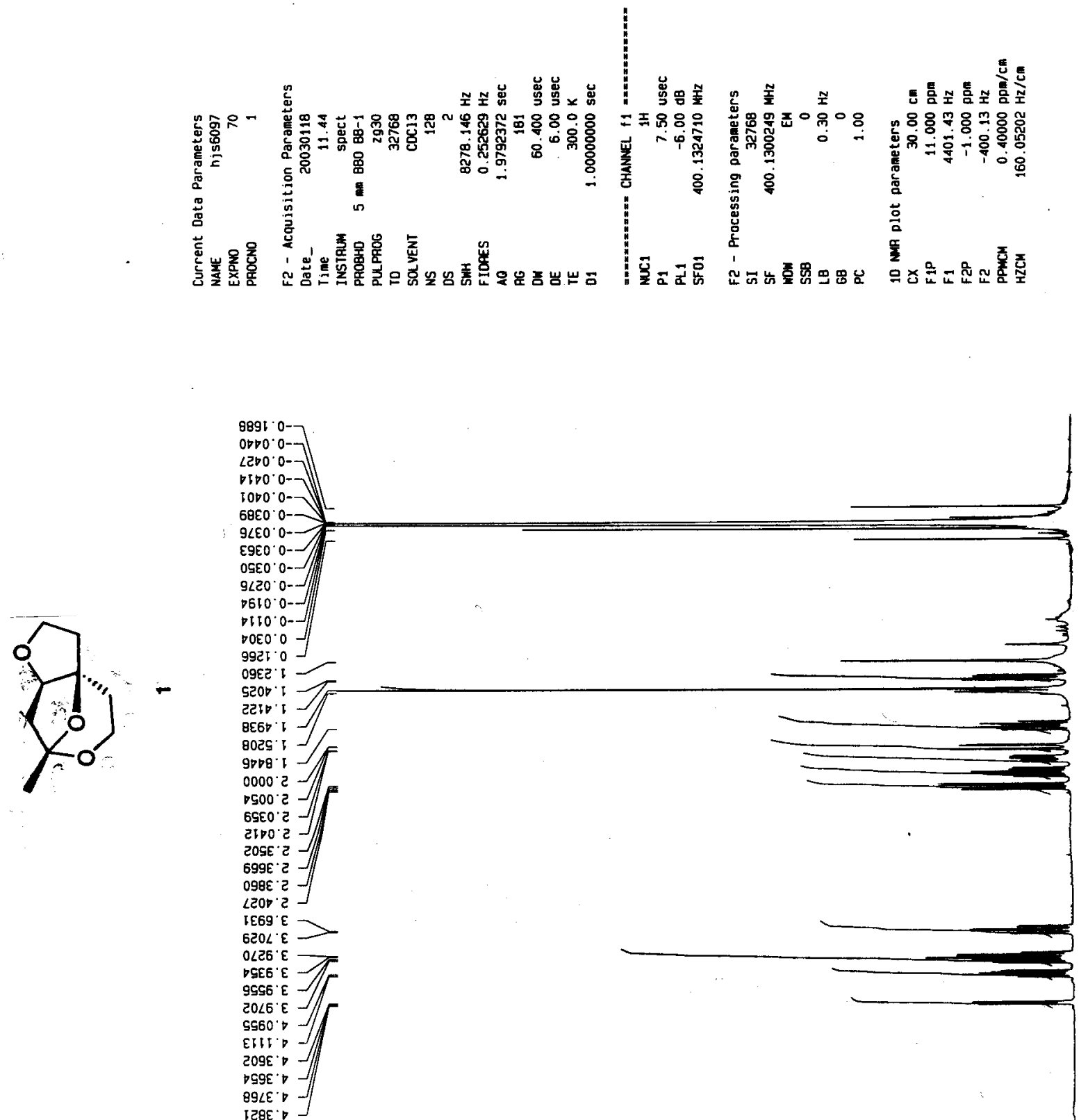

$85 \angle 6^{\circ} \cdot$

$\angle$ GEZ $L$

$9682^{\circ} L$

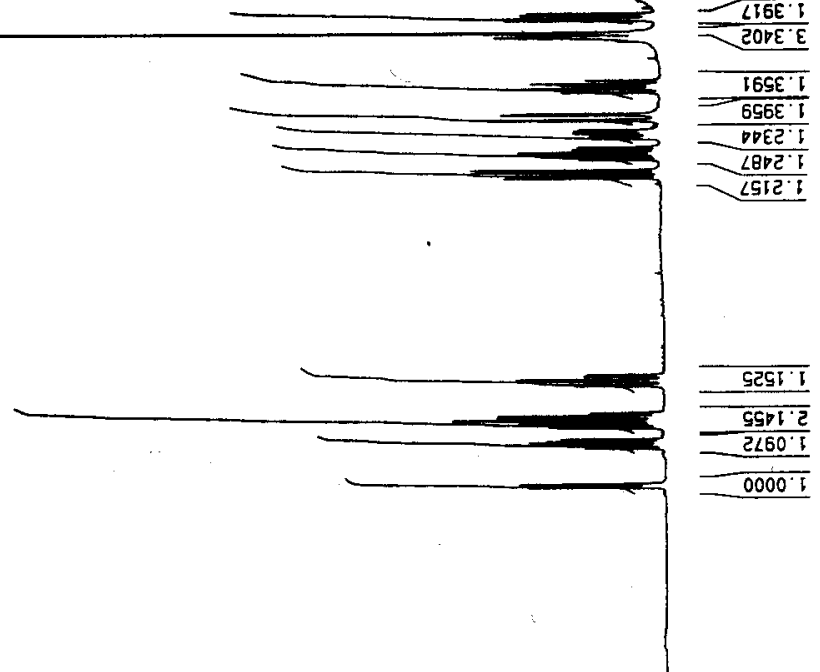




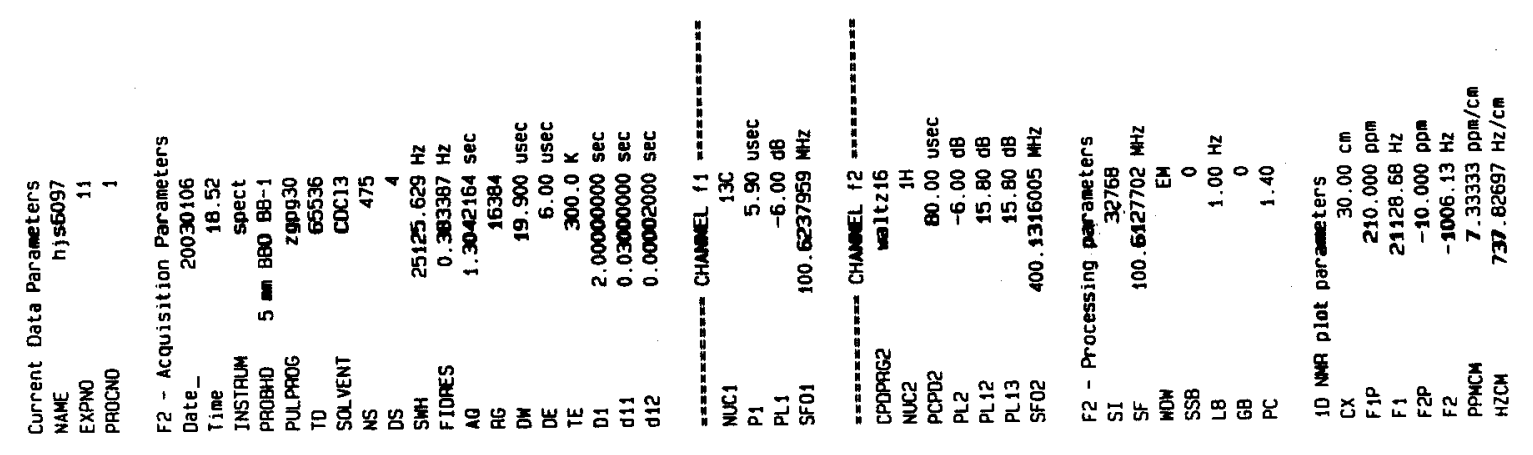

$610.0-$

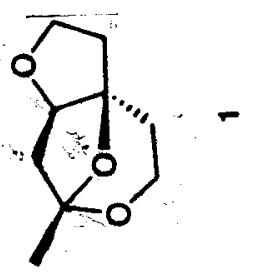

CEC DC -
OO9. OE-
OLO'9E-
$\angle D \angle D O-$

E6อ' 19

टSL'B9 -

$\nabla 89.9 L$

$100 \%$

GIE' $\angle L$

BEV $\triangle 8$

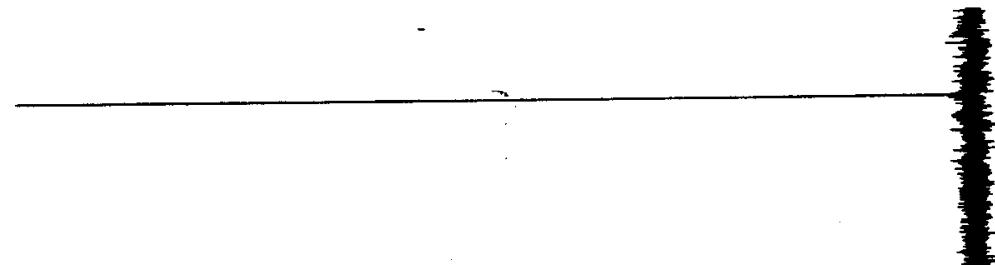

$56 b^{\circ} 16$

$897 \cdot 801-$ 

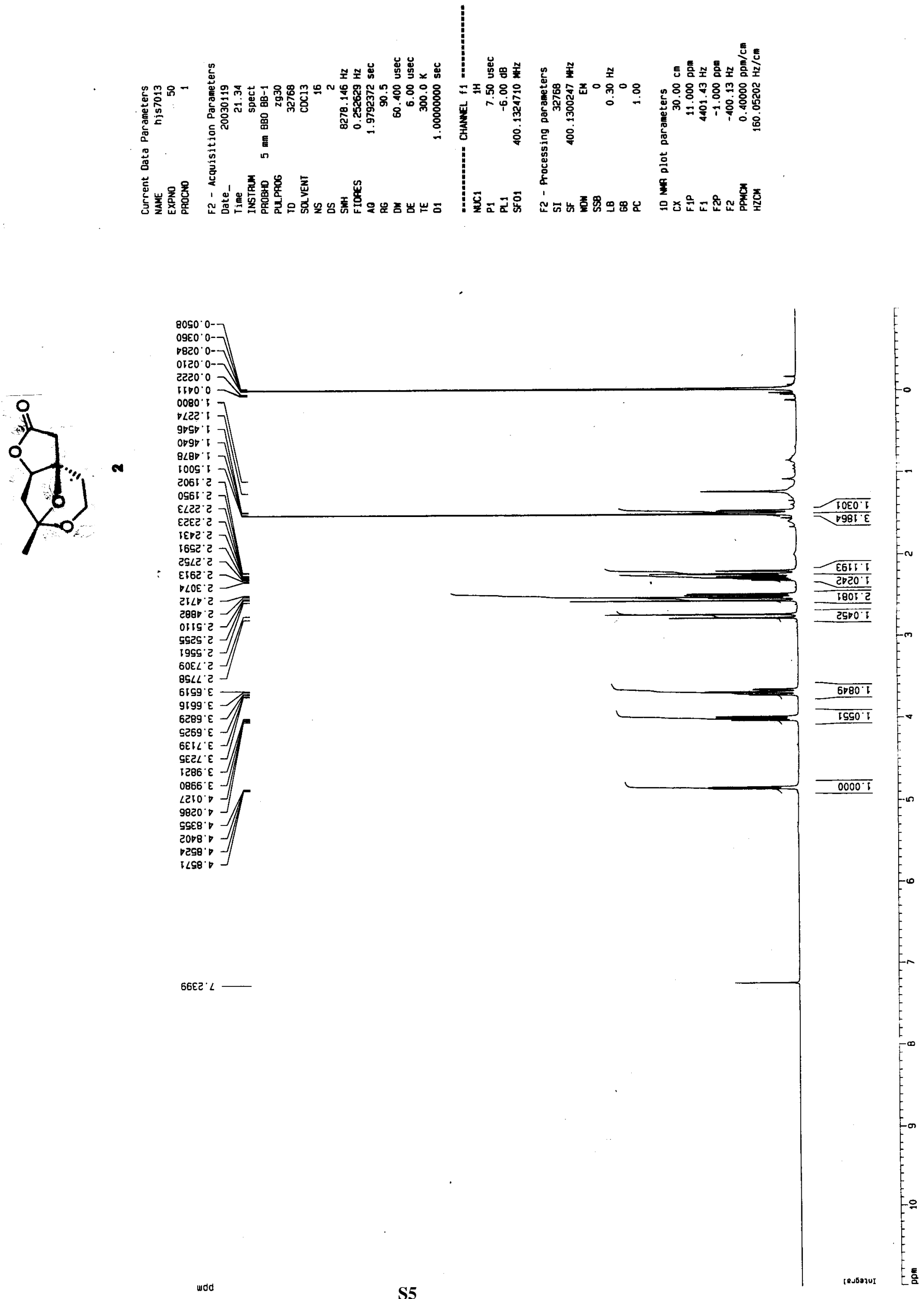

$a$ 

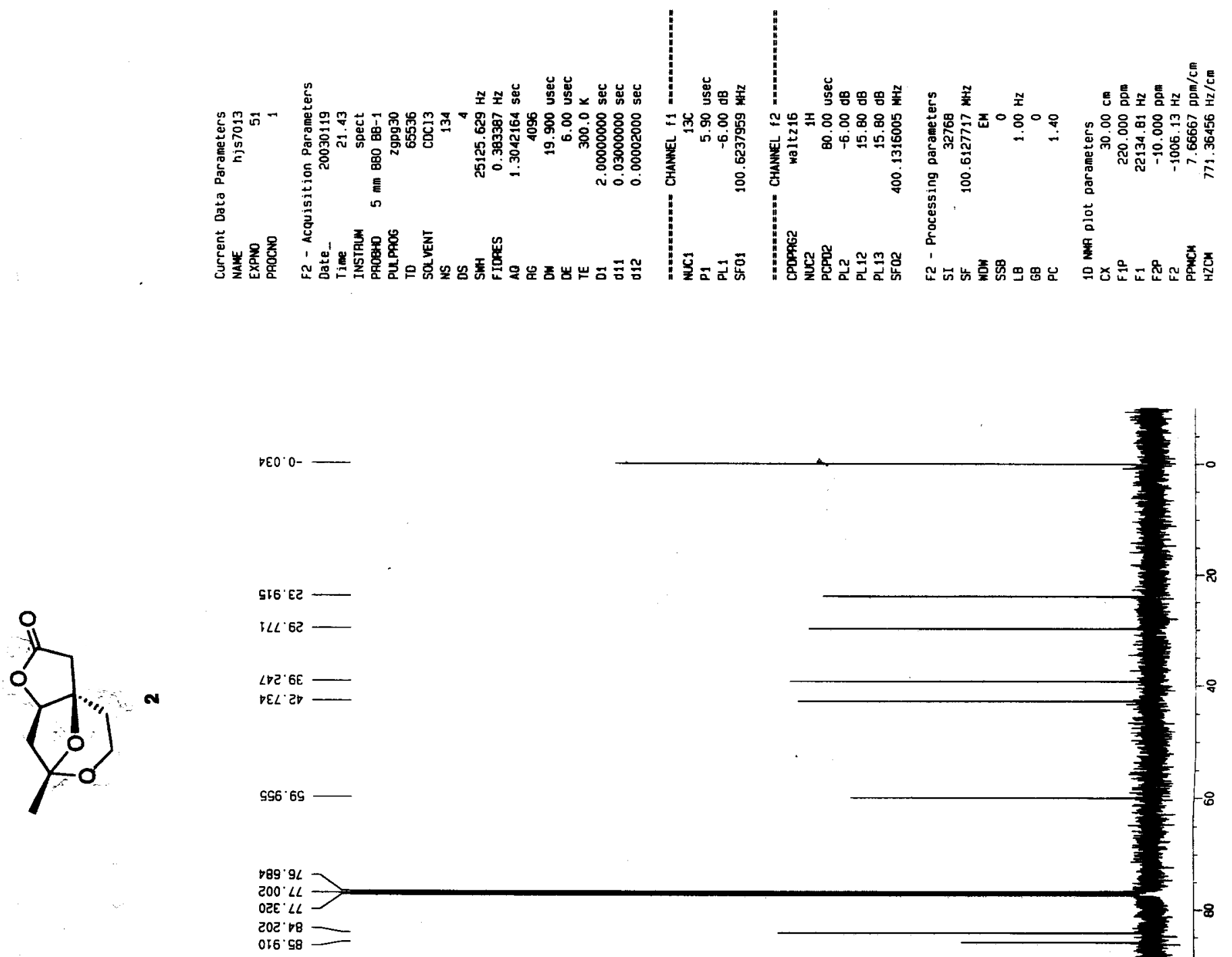

จEO.0-

$I \angle L ' \angle O T-$ 

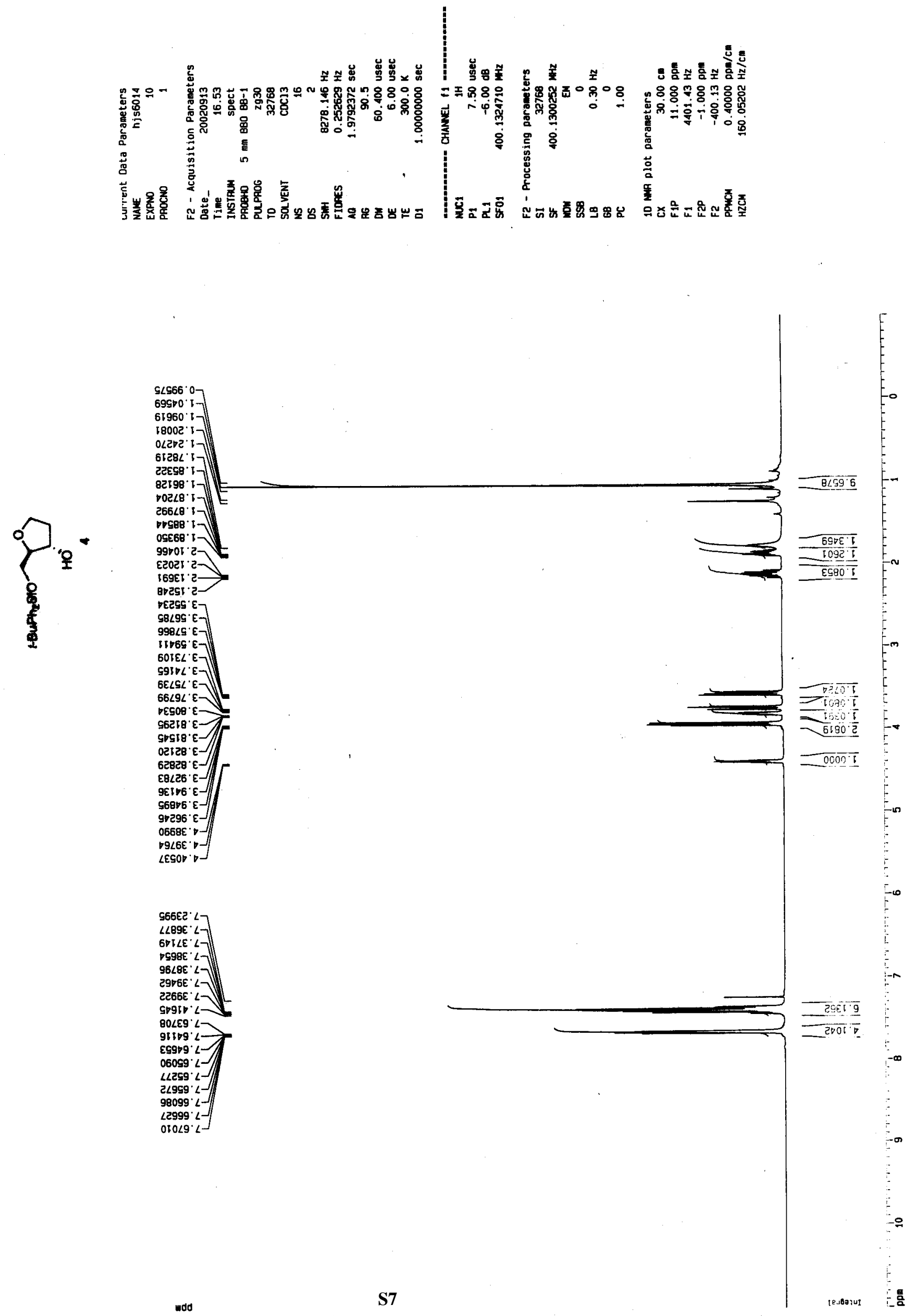


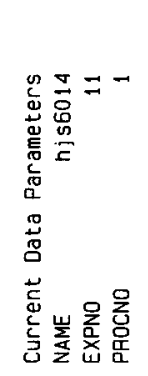

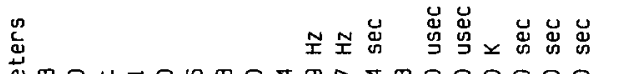

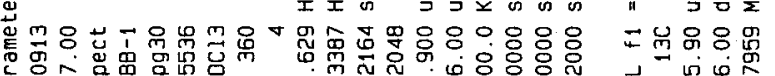

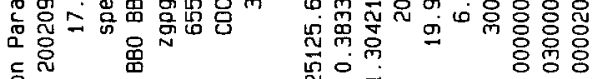

吉

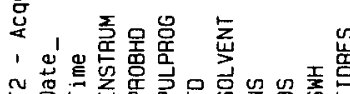

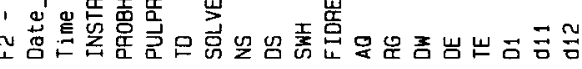

ES6โ 6 I

$6128 \cdot 92$

टOEQ $\nabla E$
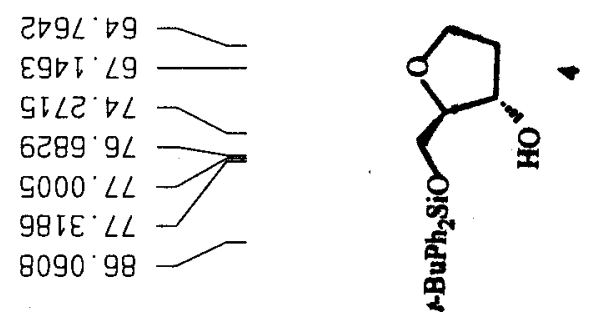

$E 9 Z \angle \angle Z I$

$86 E \angle \angle C T$

$\angle 0 G \angle$ GCL

$09 \angle L 625$

8टGI EE -

GIट己 $E E$.

6L2G'GE:

E89G $5 E$ -
巡

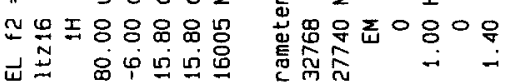

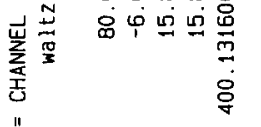

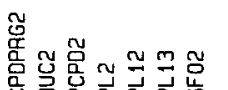

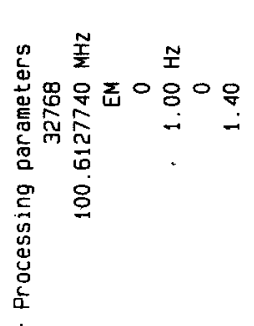

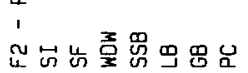

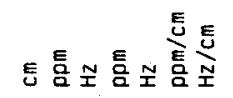

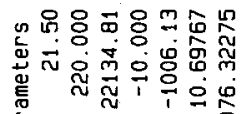

高 总

㕸

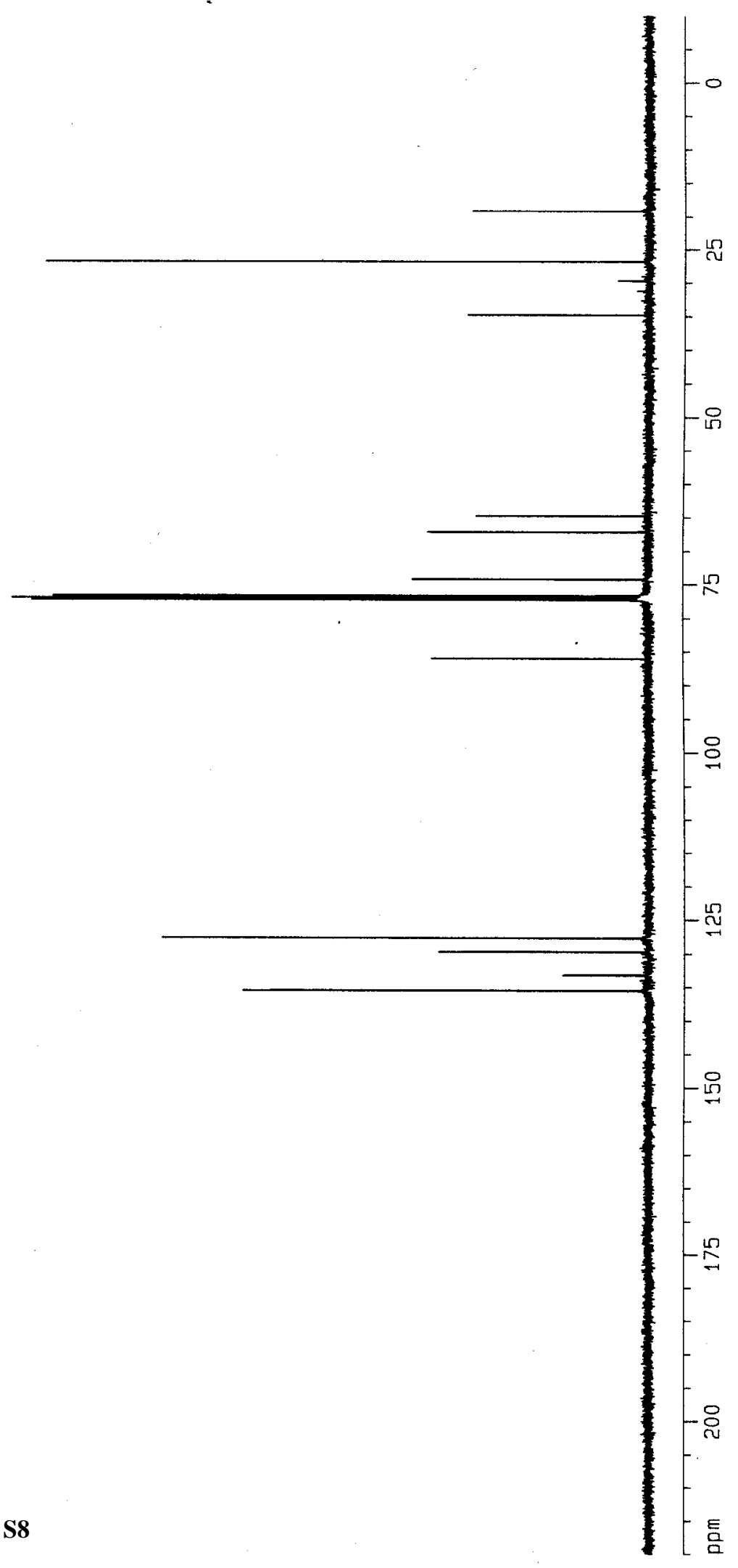



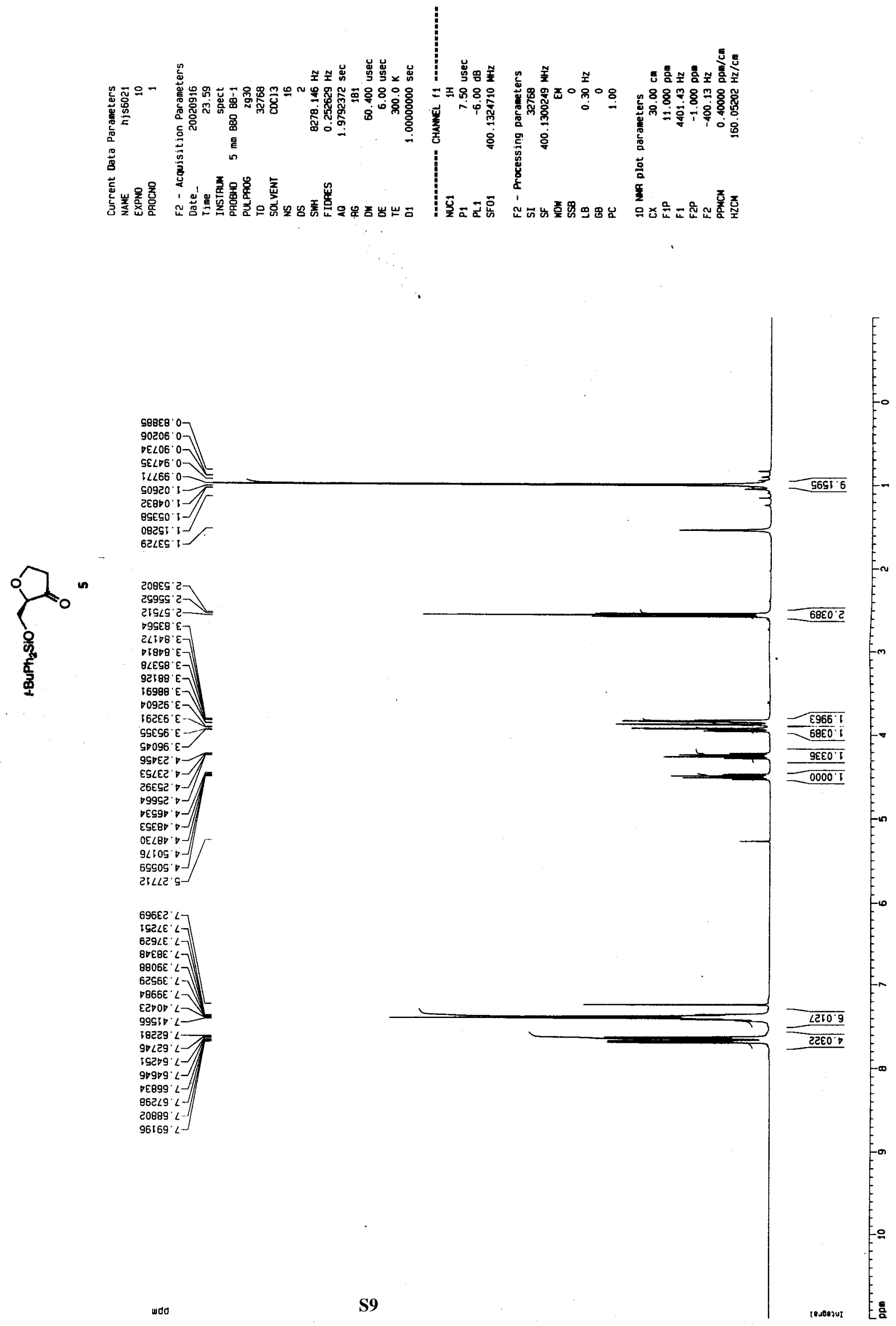


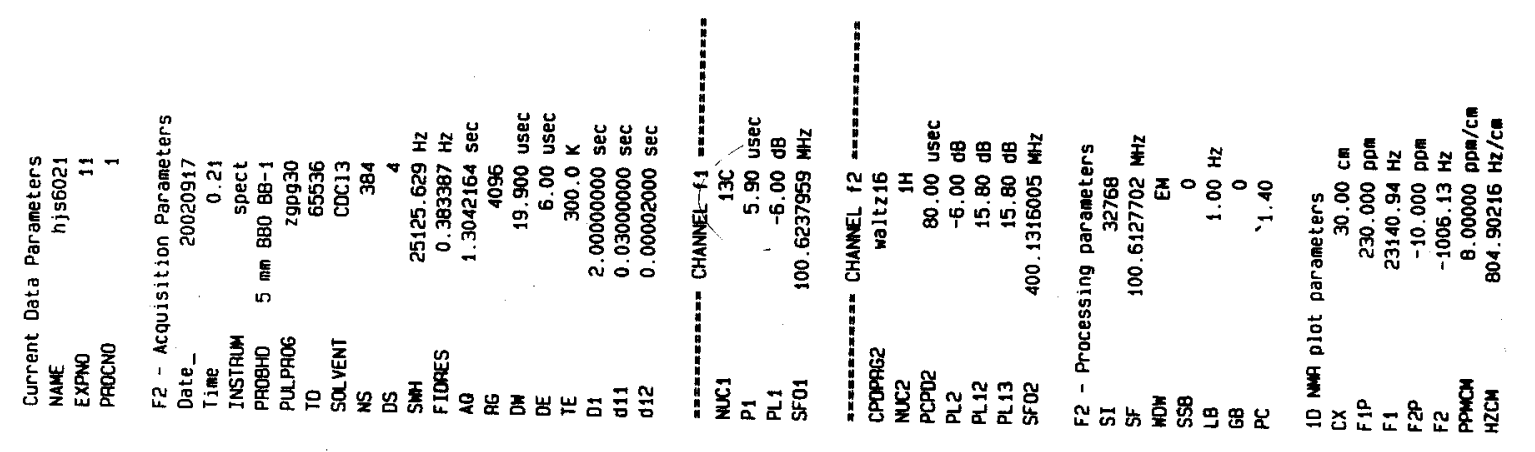

E95.65-

$\varepsilon \angle 9 \cdot 92$

PED $\angle \varepsilon=$
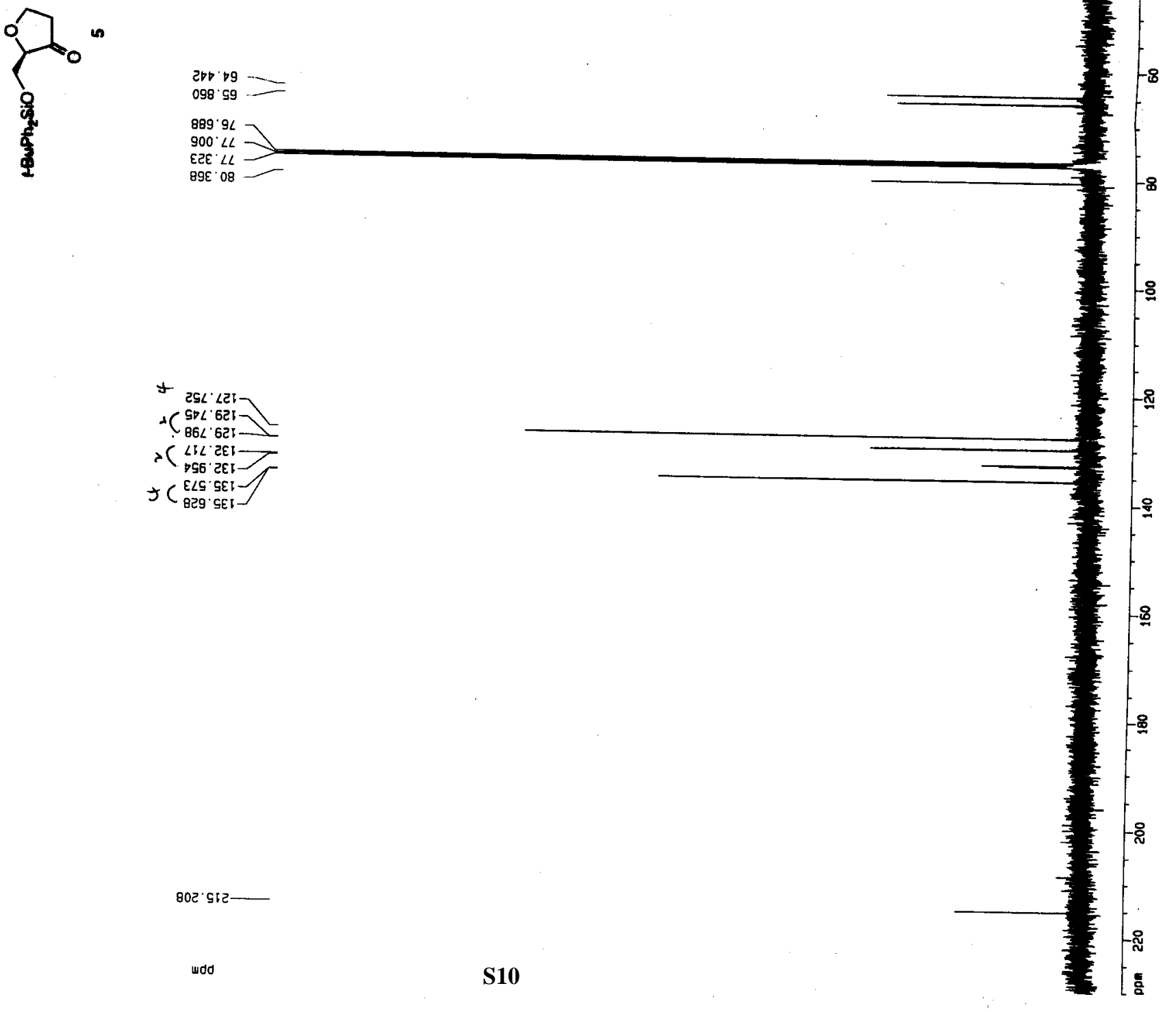

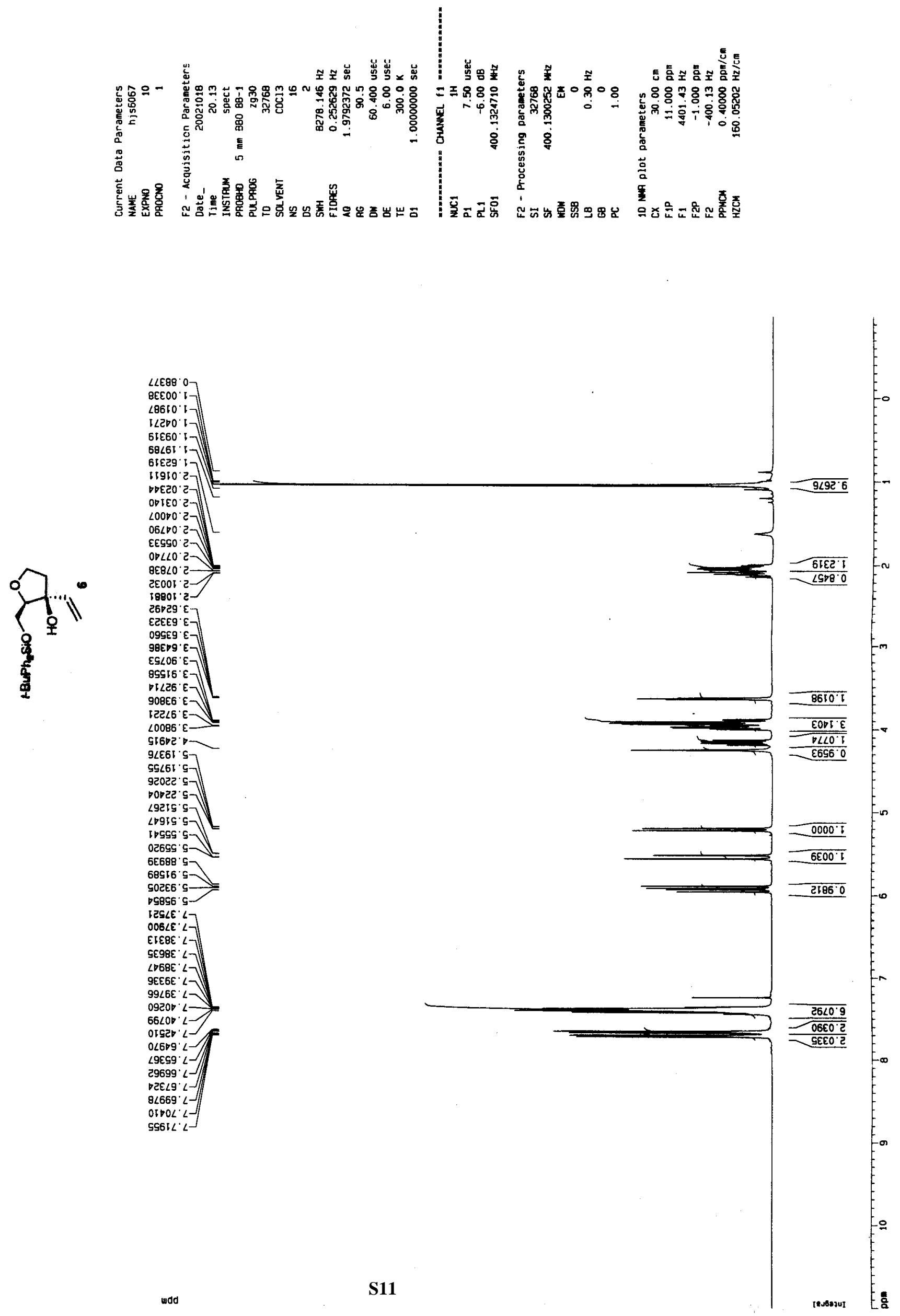


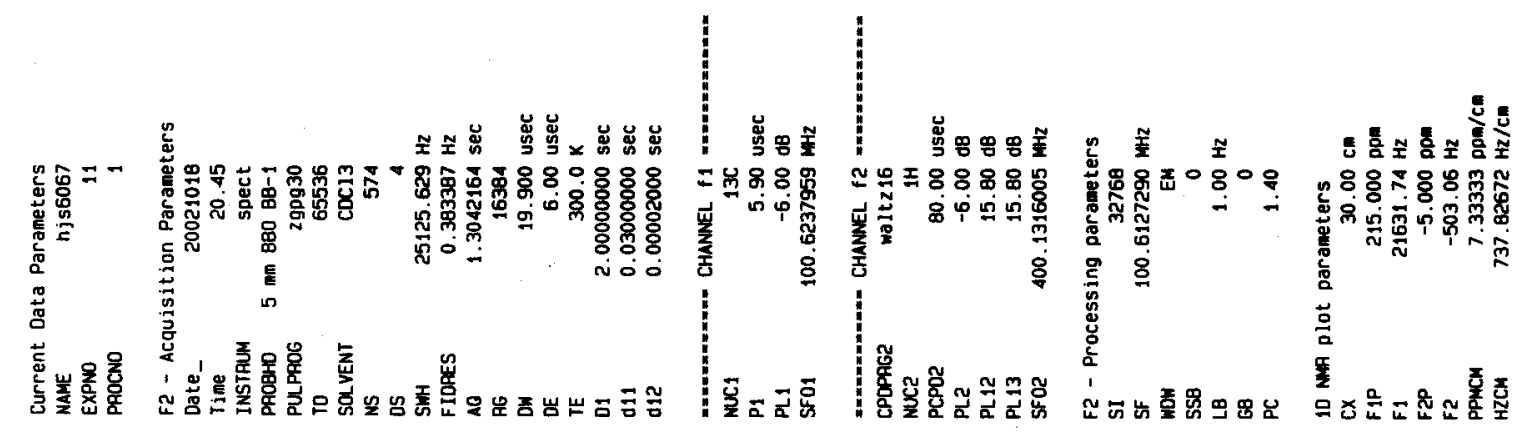

505.61

$20 I^{\circ} \angle 2$

EE6 ' it -

$\angle E O ' E 9$

$\angle 6 E \cdot \angle 9$

$\angle 21 \cdot \angle L>$

$29 L \cdot \angle L=$

$29 L 2$

I $198 \mathrm{CB}$

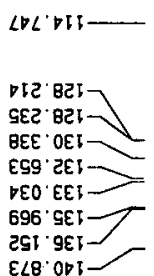



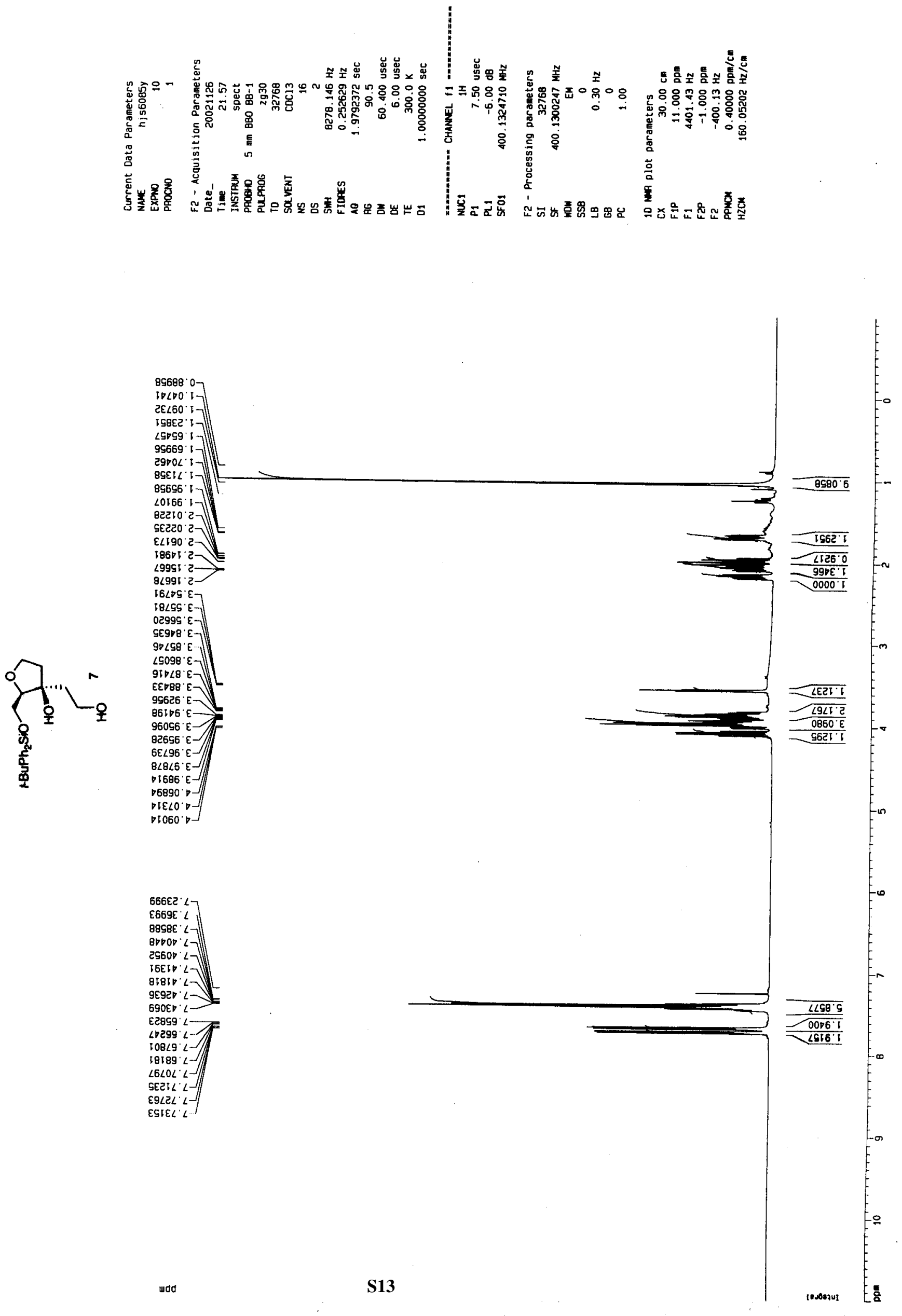

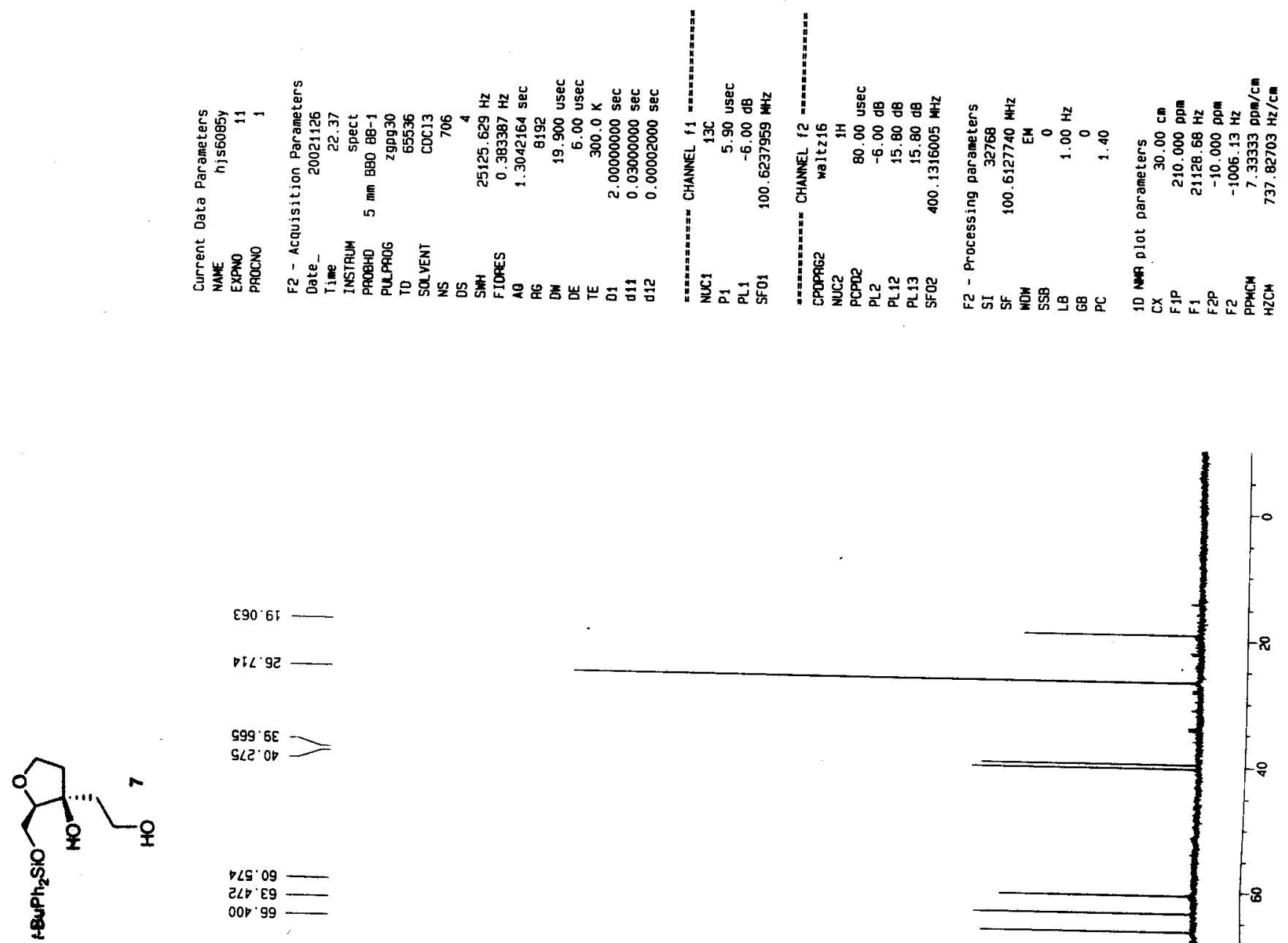

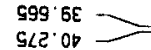
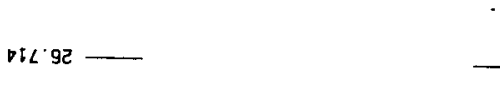

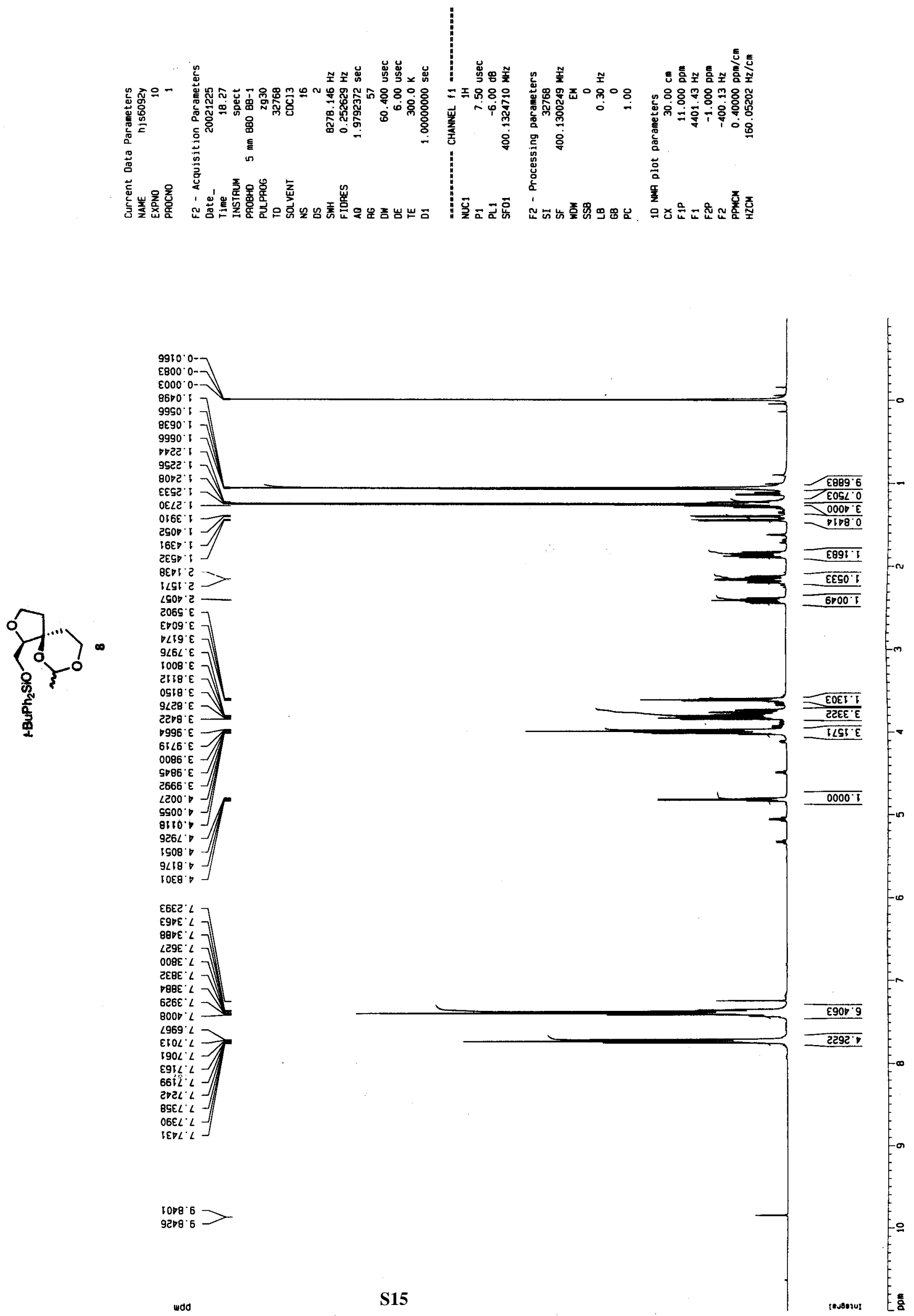


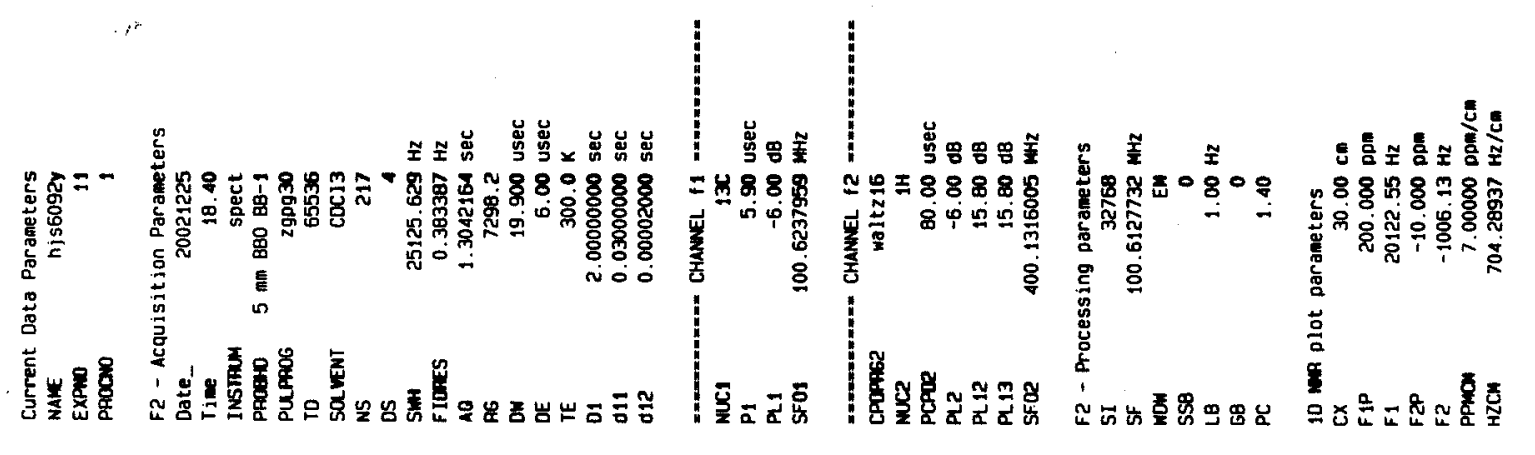

$920.0-$

$915 \cdot 615-$
$-15 \div$

128.92

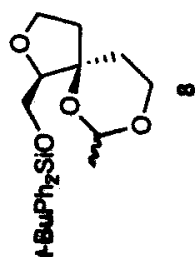

$298 \cdot 92>$

59t.' IE -

E6E' $D E$

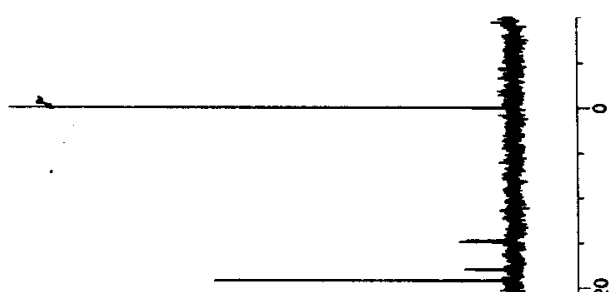

$682 \cdot 29-$

$992 \cdot 19$

$182 \cdot 99$

EBg. $9 L$

$100 . \angle L$

5IE' $\angle L$

9टE' 18

SE2 $\angle 8$

$2 \nabla 6.85-$

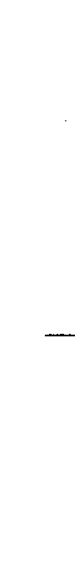

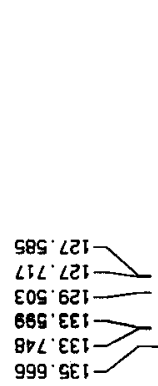



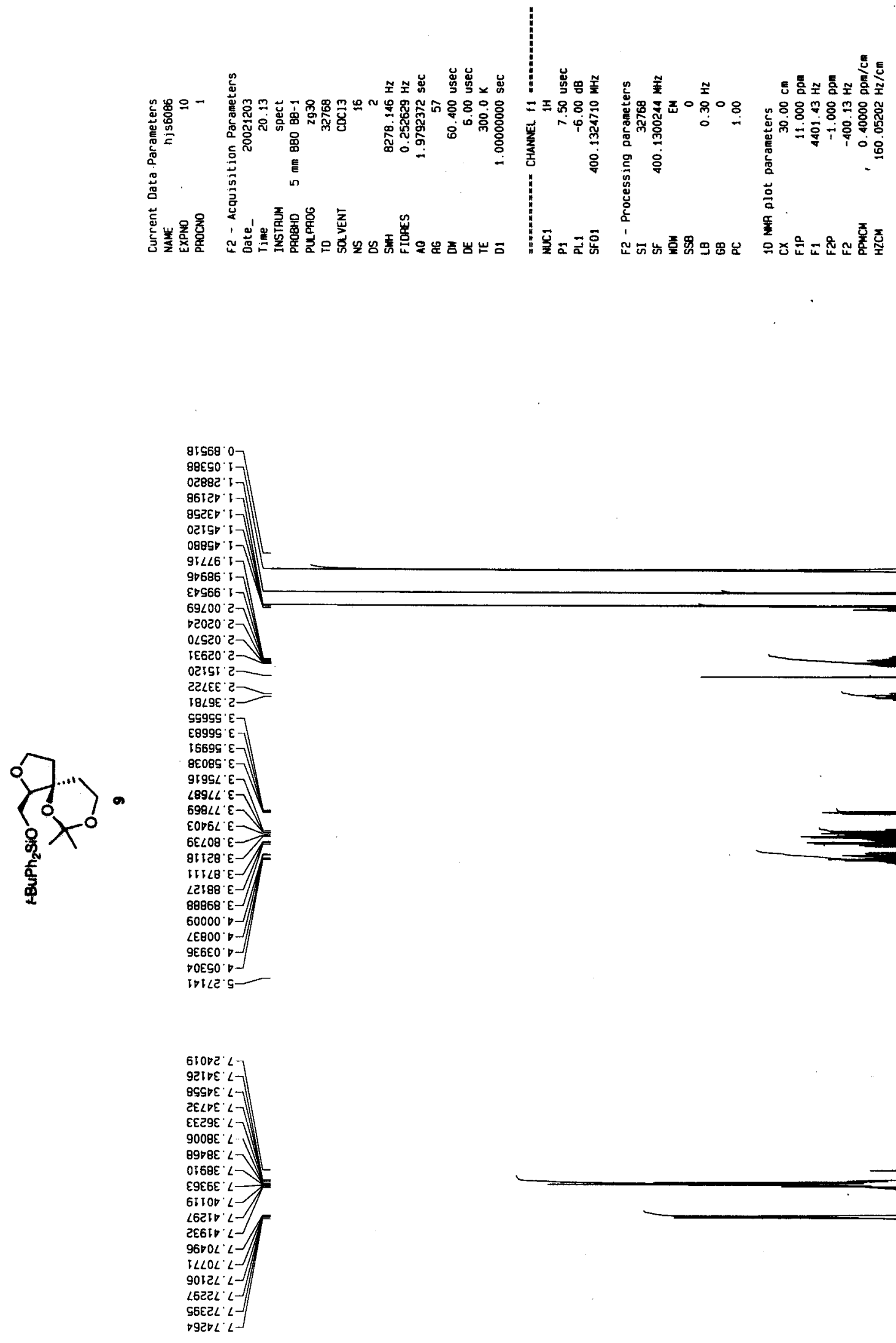

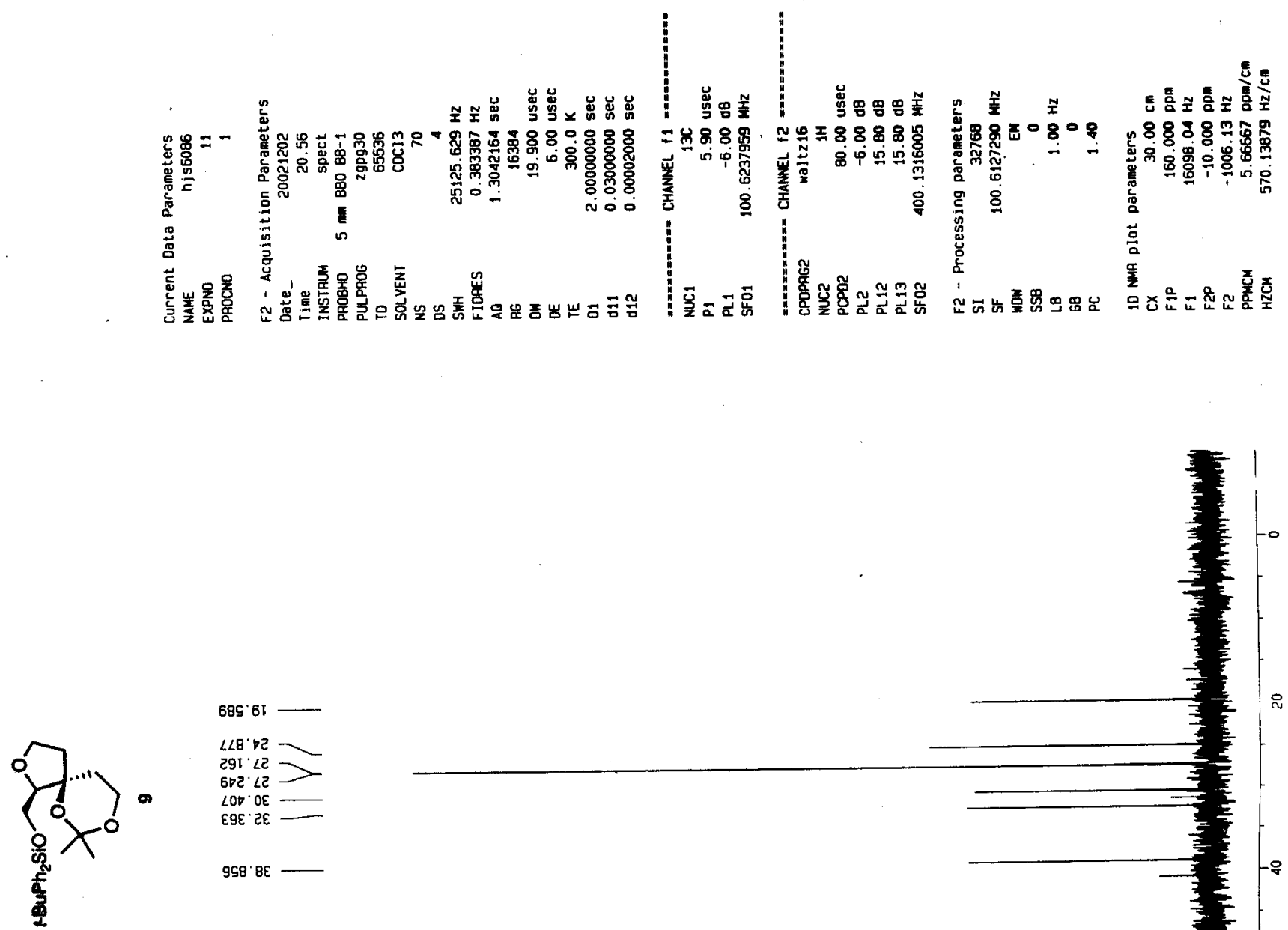

$\angle 98^{\circ} \angle 9^{-}$

960'E9 -

186. 99

O2: $\angle L$

BED $\angle L$

6SS. $6 L$

19288

$E \varepsilon \angle B S-$

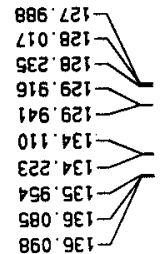

$860.961-$ 

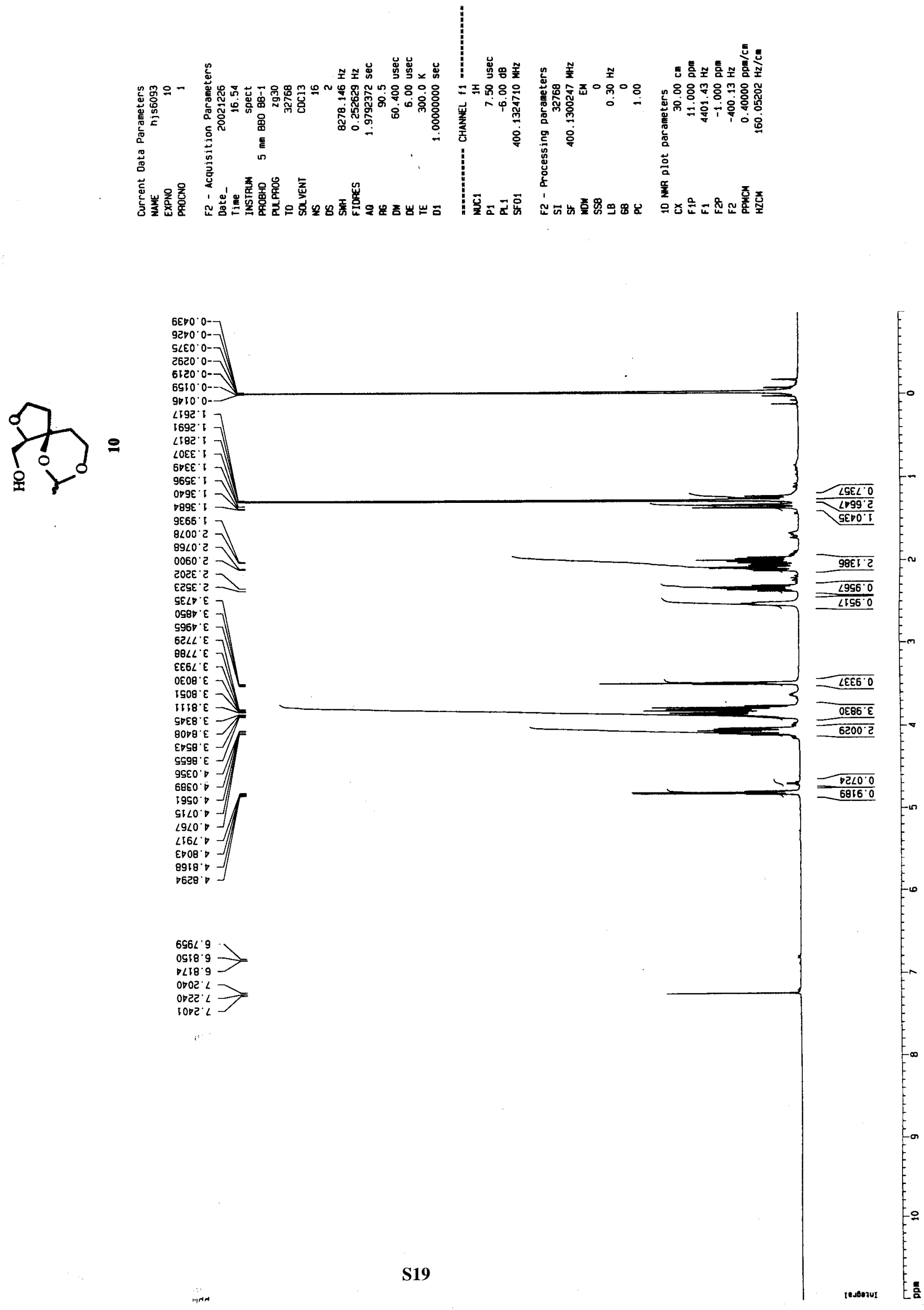


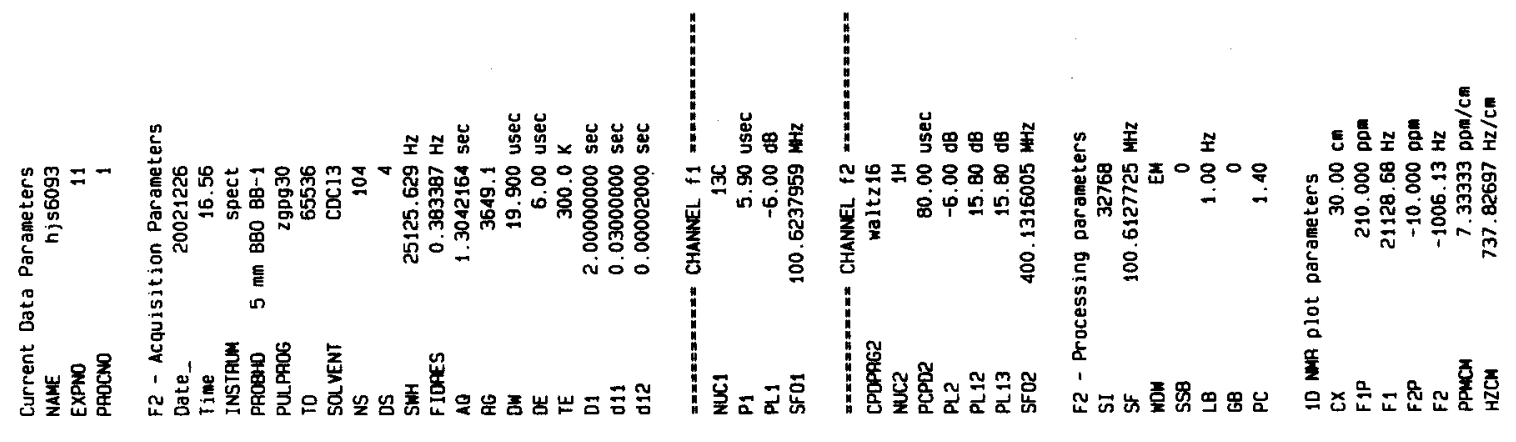

$\varepsilon 682 \cdot 52$

OOOE टE-

จอเБ'

$y_{1}=$

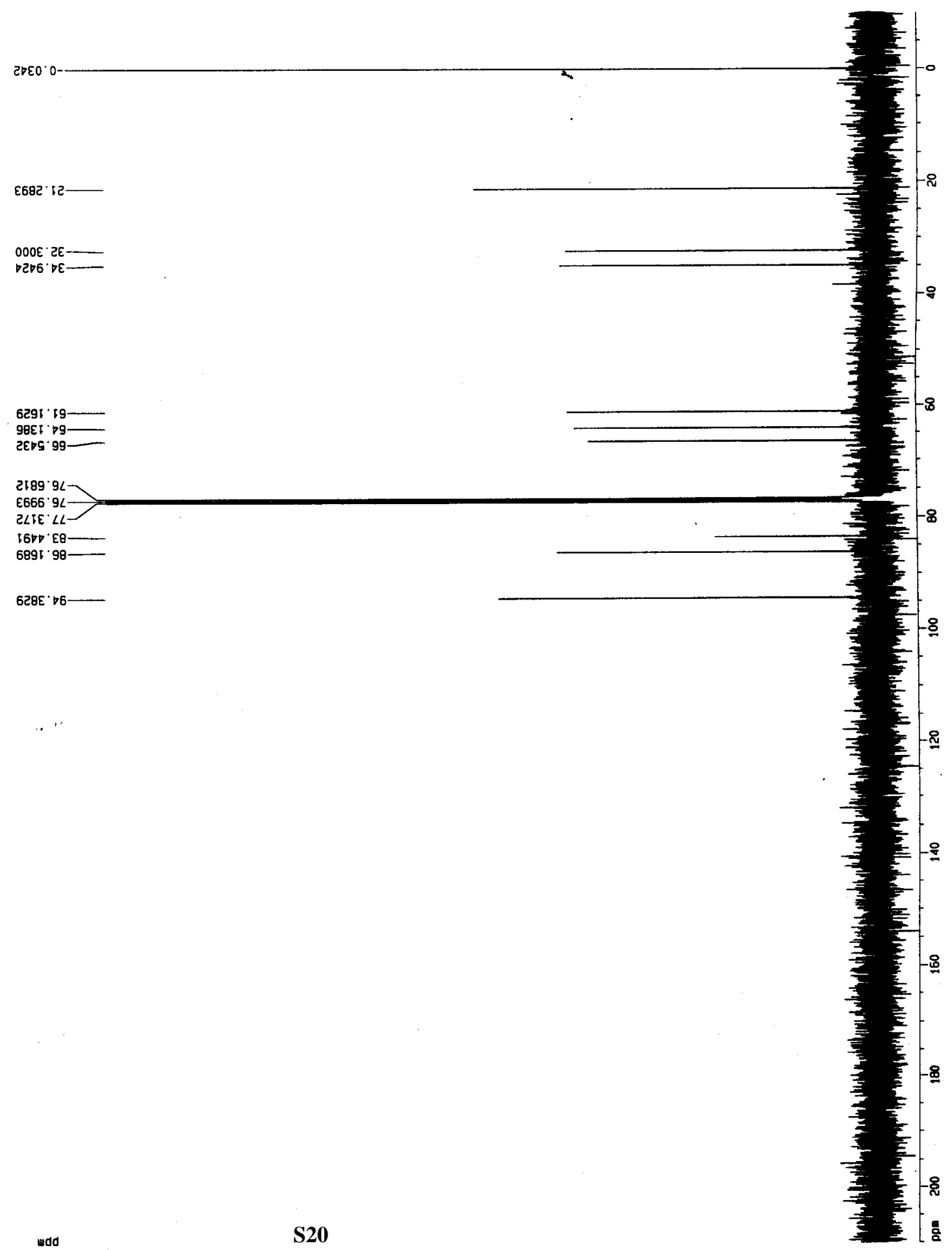



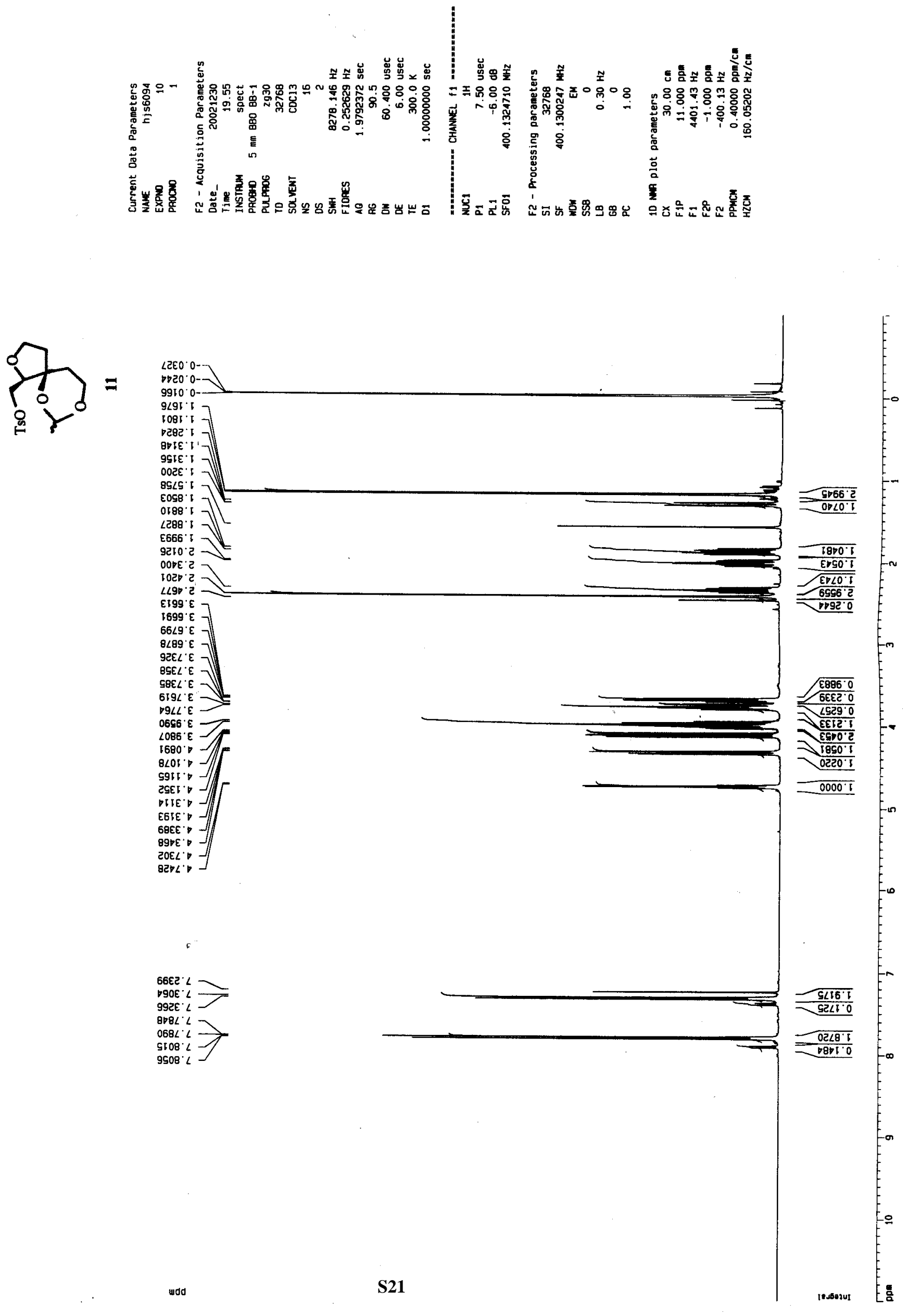


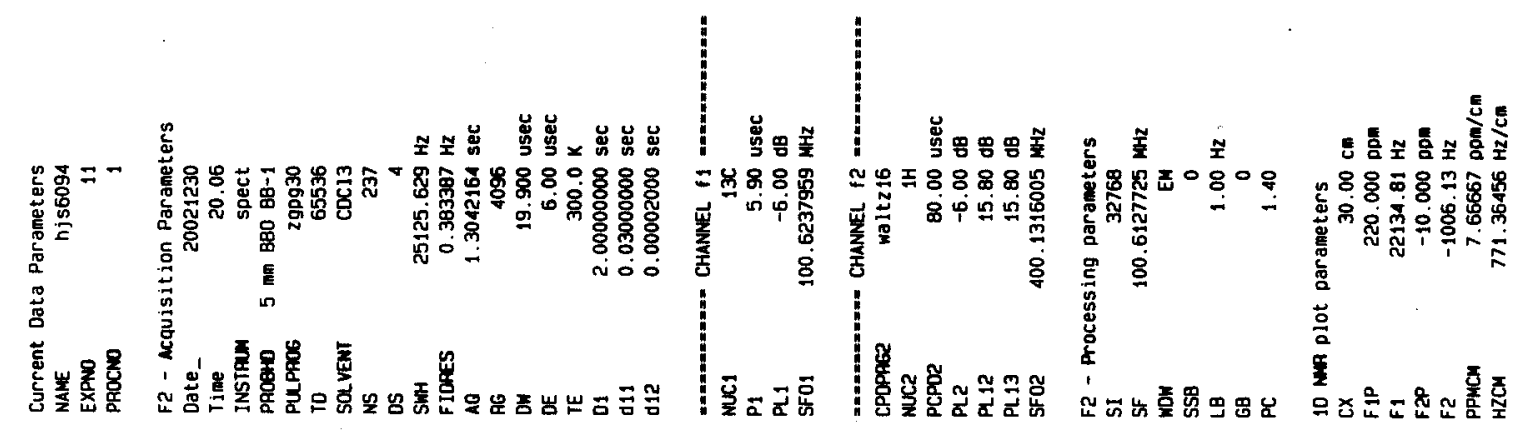

$7_{0}^{0}=$

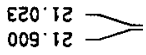

เรE' $1 \varepsilon=$

Ste

S66. 109

$280.69=$

5 $\angle 9 ' 9 \angle$

266.94

oIE $L L$

OE6 $18=$

150.86

296 ㄴㄷ

$896.621=$

ISI 'EEः-

$629 \cdot P D I-$ 

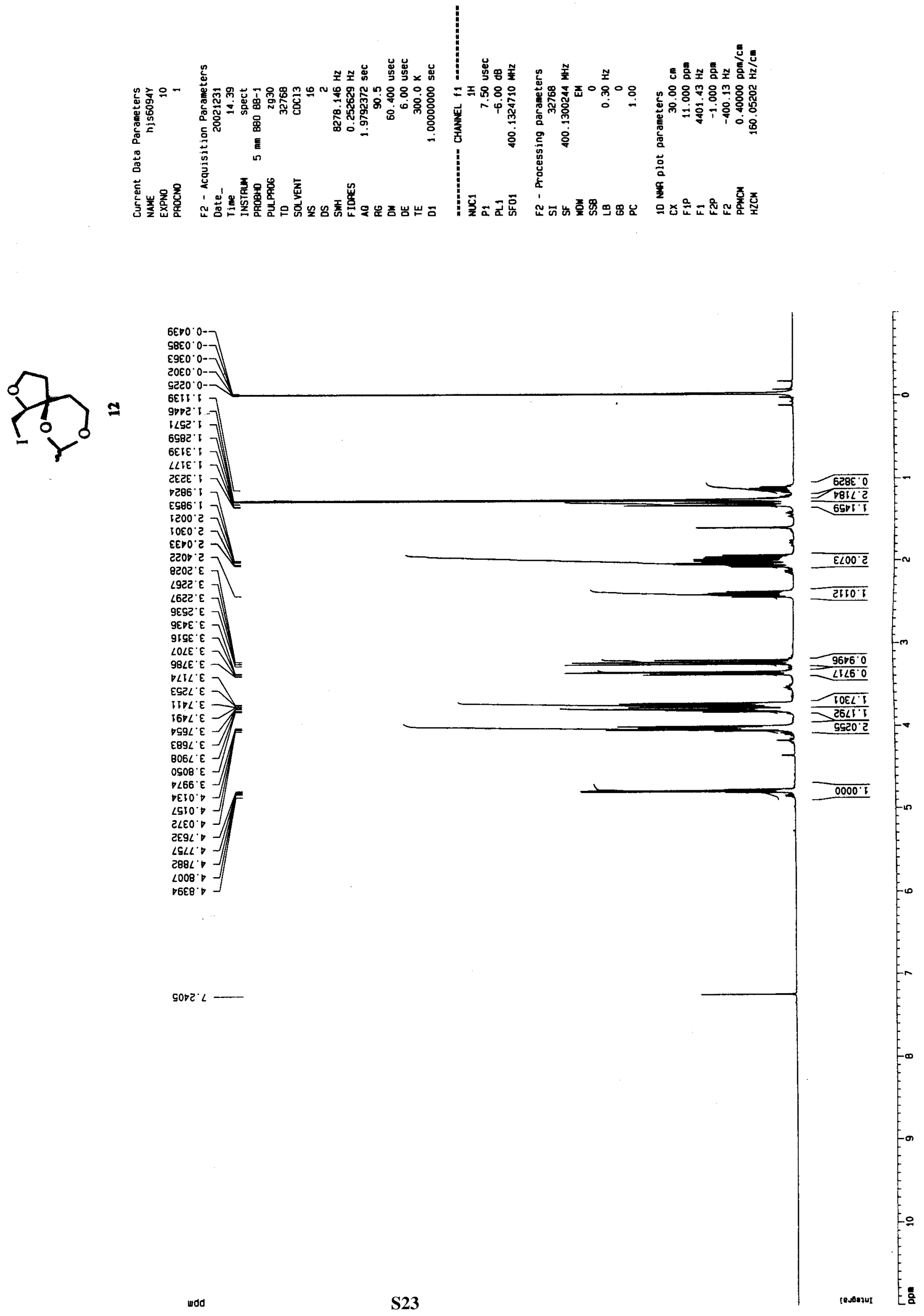


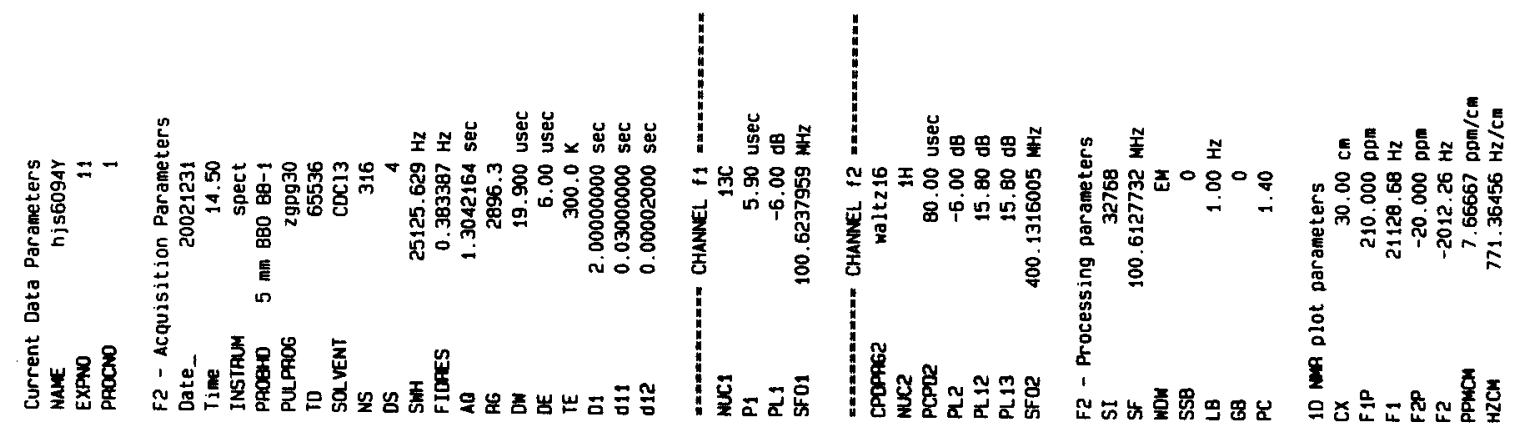

9270.0-

$\varepsilon \angle 06^{\circ} \mathrm{Z}-$

DGEI IL-

$y^{0}=$

0618. IE- -

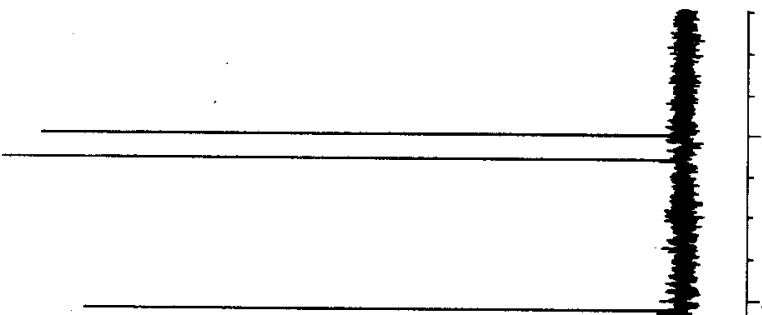

6010.09

0096.59

$8009^{\circ} 9 L$

$\checkmark 866^{\circ} 94$

GStE $\angle L>$

$89 \angle 9^{\circ} \angle 8$

$\checkmark D \nabla \cdot \nabla 6$ 

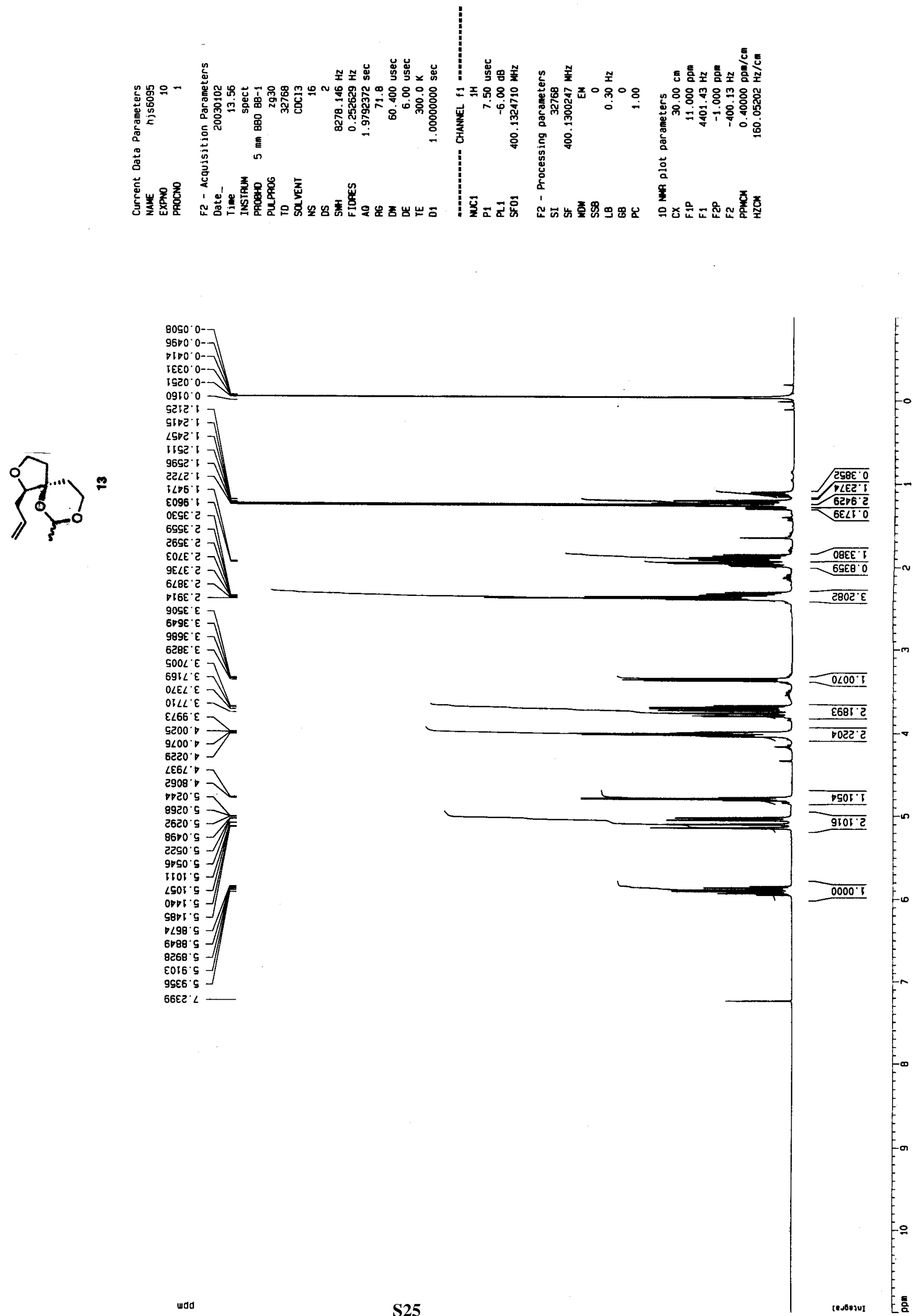


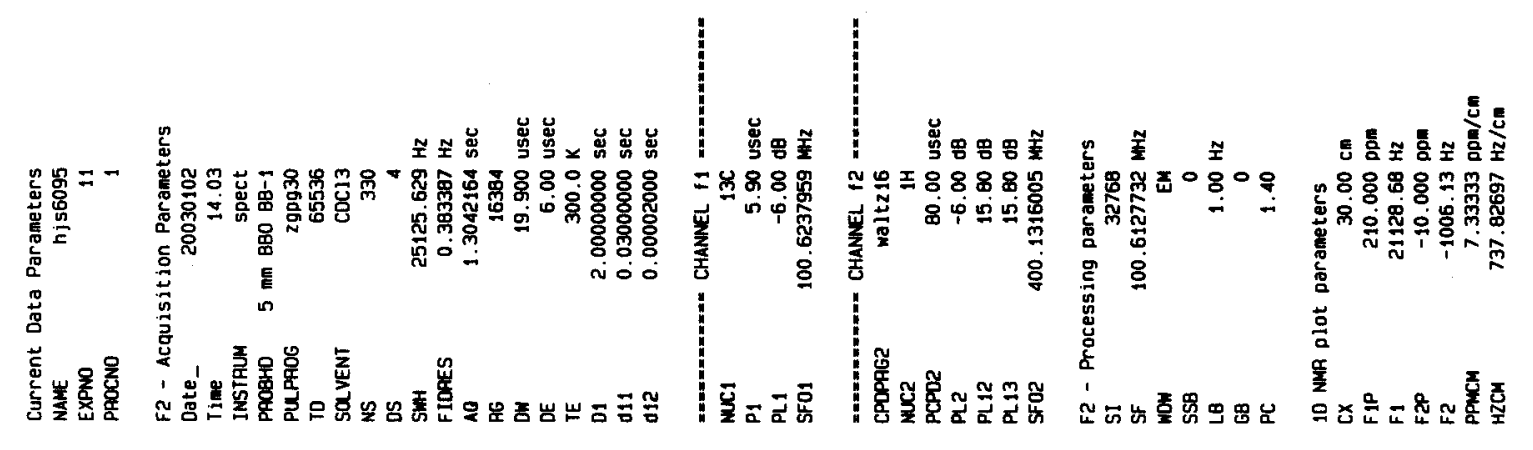

$950.0-$

60212

DEP IE $~$
CIG $2 E-$

$\sum_{3=0}=$

$\nabla 99^{\circ} \nabla \varepsilon-$

$902^{\prime} \triangleright 9-$

858.99

$089 \cdot 9 L$

$866.9 L$

जเE $\angle L$

OOZ' IB -

499.98

926.86

26 '91:-

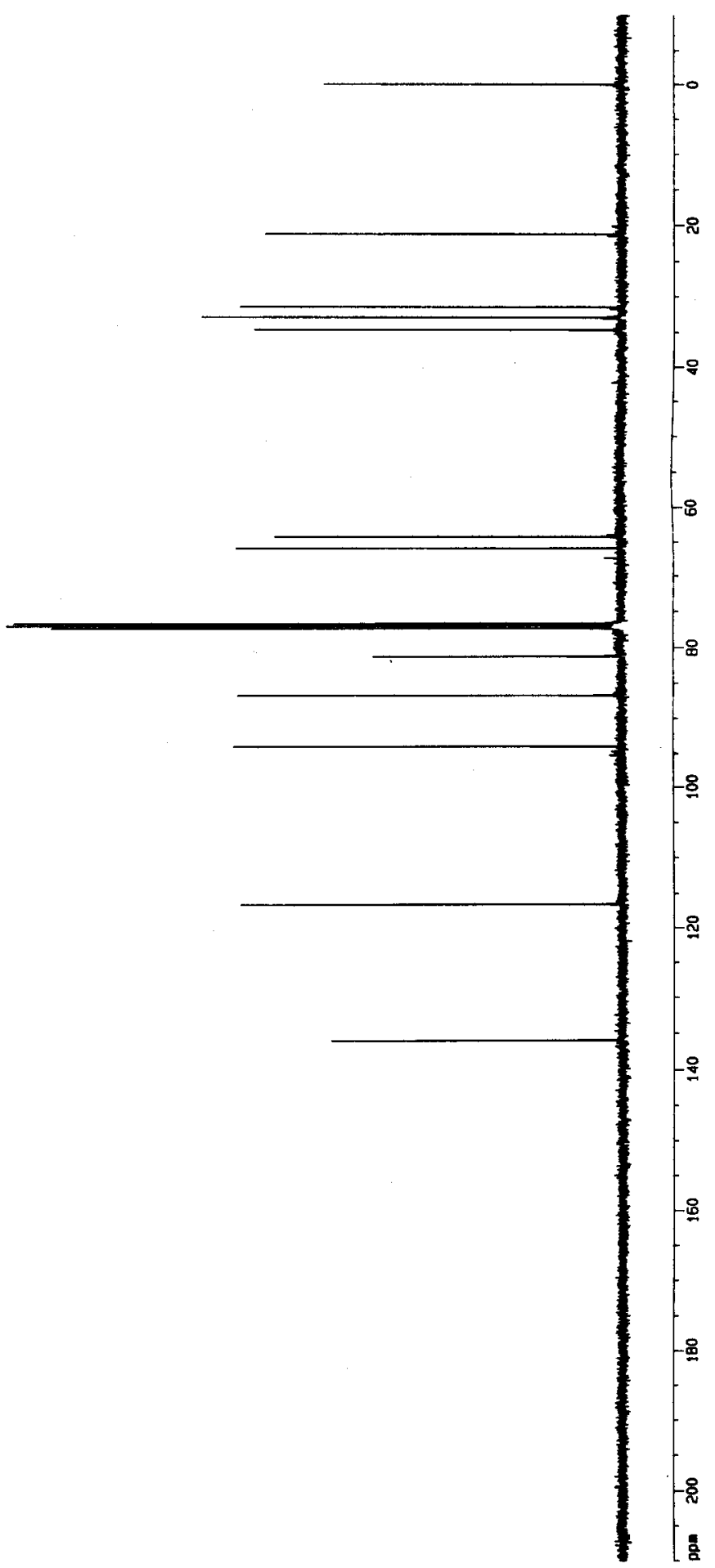



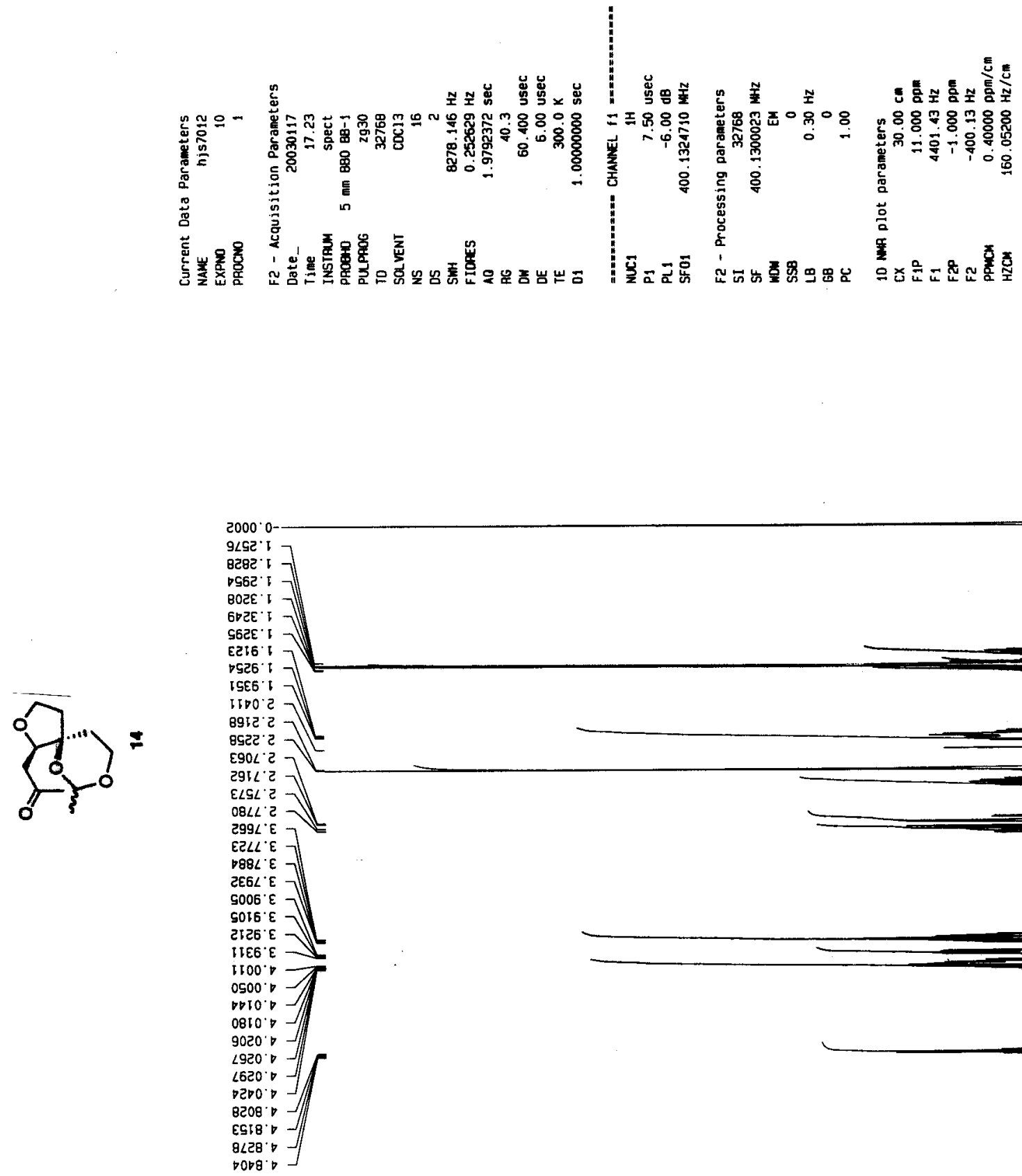


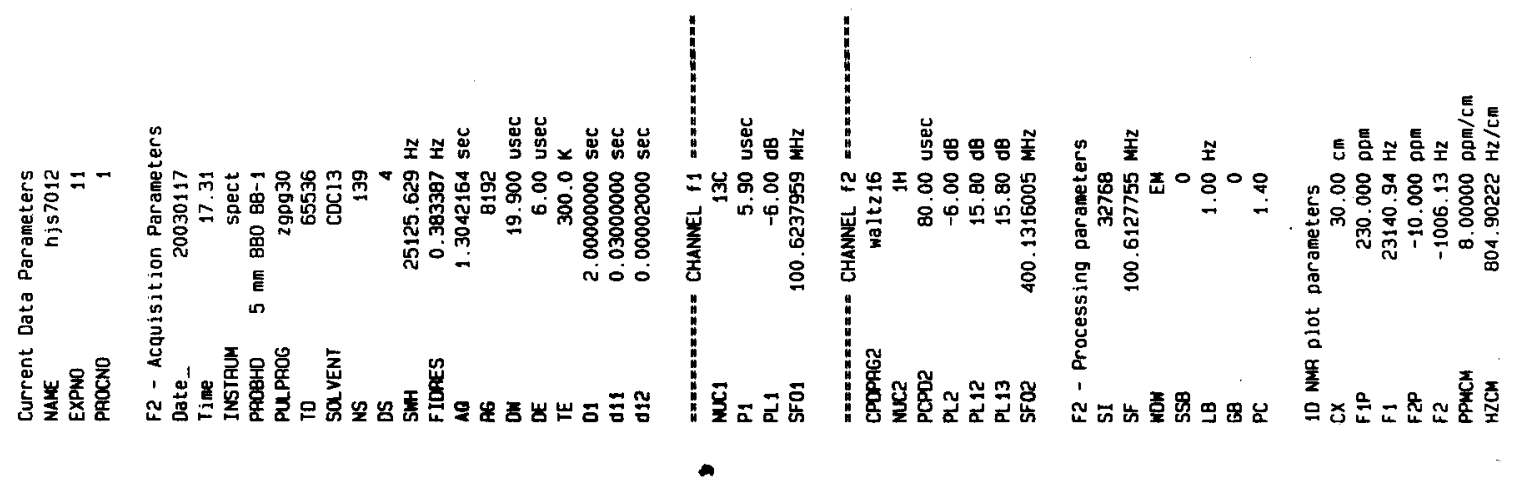

$1010-$

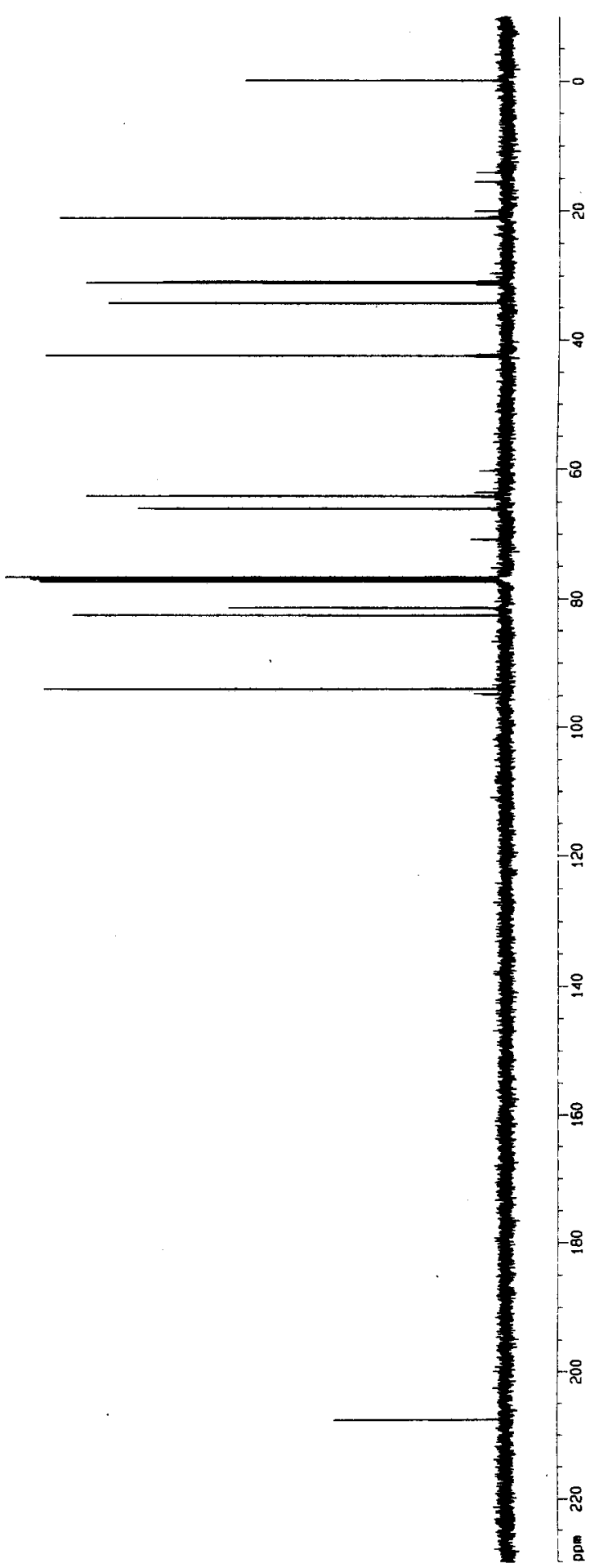



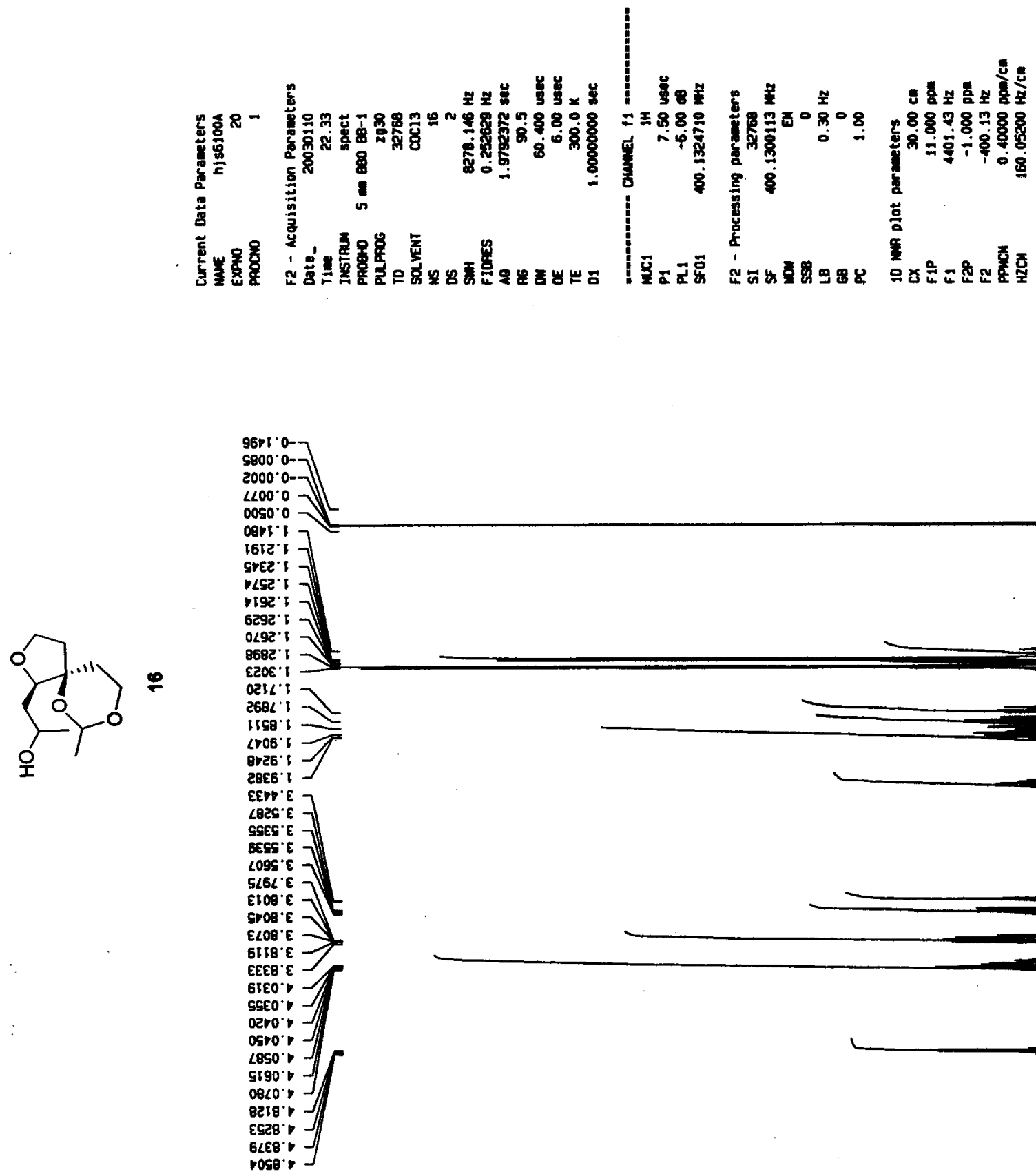

IELC L - 


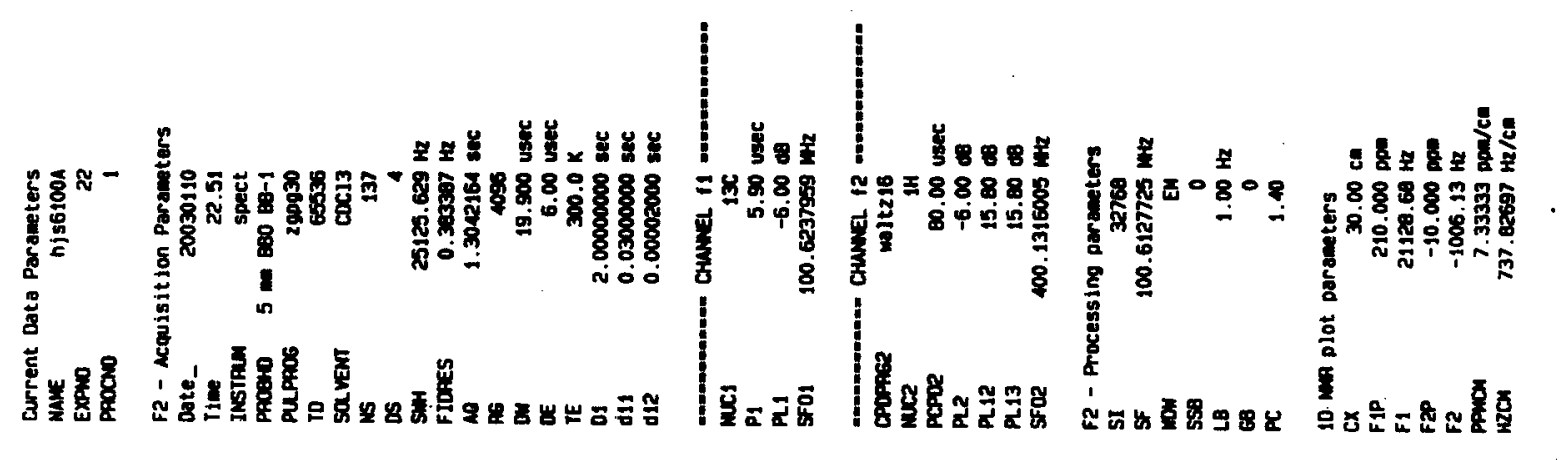

$\angle 6 r 0^{\circ} 0$

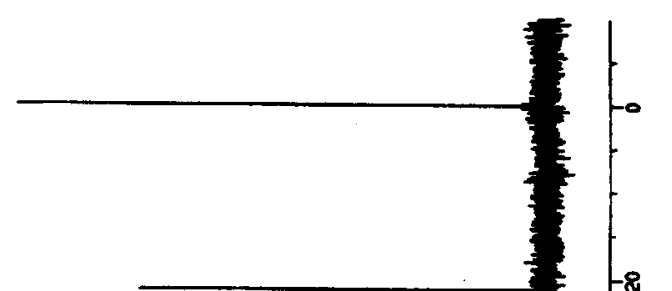

9095' 12

TEIt IE-

$\angle 926^{\circ} \mathrm{EE}-$

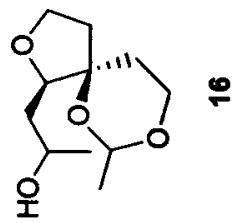

WWE. $9 \varepsilon$

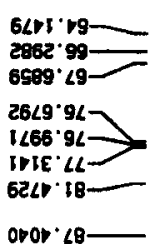

$\operatorname{Ir} \angle 6 \cdot 86$ 